\title{
Comparison and Continuity of Wick-type Star Products on certain coadjoint orbits
}

\author{
Chiara Esposito*, \\ Dipartimento di Matematica \\ Università degli Studi di Salerno \\ via Giovanni Paolo II, 123 \\ 84084 Fisciano (SA) \\ Italy \\ Philipp Schmitt ${ }^{\dagger}$ \\ Department of Mathematical Sciences \\ University of Copenhagen \\ Universitetsparken 5 \\ DK-2100 København $\varnothing$ \\ Denmark \\ Stefan Waldmann \\ Institut für Mathematik \\ Lehrstuhl für Mathematik X \\ Universität Würzburg \\ Campus Hubland Nord \\ Emil-Fischer-Straße 31 \\ 97074 Würzburg \\ Germany
}

\begin{abstract}
In this paper we discuss continuity properties of the Wick-type star product on the 2-sphere, interpreted as a coadjoint orbit. Star products on coadjoint orbits in general have been constructed by different techniques. We compare the constructions of Alekseev-Lachowska and Karabegov and we prove that they agree in general. In the case of the 2 -sphere we establish the continuity of the star product, thereby allowing for a completion to a Fréchet algebra.
\end{abstract}

${ }^{*}$ chesposito@unisa.it

${ }^{\dagger}$ philippschmitt@math.ku.dk

${ }^{\ddagger}$ stefan. waldmann@mathematik. uni-wuerzburg. de 


\section{Contents}

1 Introduction $\quad 2$

2 Preliminaries 4

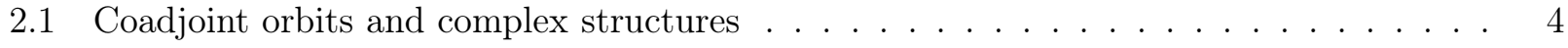

2.2 A construction by Karabegov . . . . . . . . . . . . . . . . . . 6

2.3 A construction by Alekseev-Lachowska . . . . . . . . . . . . . . . . . 9

3 The products of Karabegov and Alekseev-Lachowska agree 11

4 First convergence properties

5 Continuity of the star product on $\mathbb{S}^{2} \quad 16$

5.1 Formulas for $\mathrm{SU}(2)$ and related properties . . . . . . . . . . . . . . 17

5.2 Continuity in the reduction and quotient- $T_{R}$-topologies $\ldots \ldots \ldots \ldots$

\section{References}

\section{Introduction}

There are two main directions in the field of deformation quantization: formal and strict quantization. The first of these was introduced in [2] and has a rather algebraic flavour. One considers an associative product for formal power series of smooth functions on a Poisson manifold as a deformation of the commutative product of such functions, in the direction of the Poisson bracket. Existence and classification of these formal quantizations on symplectic manifolds have been understood [5, 17, 19, 33, and the general case of Poisson manifolds was solved by Kontsevich's formality theorem [26]. However, to be physically relevant formal deformation quantization lacks one important property: one cannot just plug in a real value representing Planck's constant due to convergence issues of the formal power series.

Strict deformation quantization [31,32, 35] attempts to overcome this problem by looking at fields of algebras defined for a suitable set of values for the deformation parameter. However, the situation in this context is much more unclear than in the formal setting and there are several competing definitions on what strict quantization should be. A prominent one is due to Rieffel [35, who works with continuous fields of $\mathrm{C}^{*}$-algebras. In his work, he constructs such a field out of an isometric action of $\mathbb{R}^{d}$ on an arbitrary $\mathrm{C}^{*}$-algebra. Although this setting is fairly general, important symplectic manifolds like the 2-sphere with its $\mathrm{SO}(3)$-invariant symplectic structure cannot be treated in this approach [36]. The method introduced by Rieffel, also used by Bieliavsky and Gayral in [7] for the case of negatively curved Kählerian Lie groups, relies on oscillatory integrals, which makes this approach rather technical and impossible to generalize to infinite dimensional settings (as needed when studying quantum field theories), see also the recent work [6].

Another approach tries to introduce locally convex topologies [3,4,39] in which the formal power series converge on sufficiently large subalgebras. Several examples have been studied in this area, including also infinite dimensional ones like star products of exponential type in [38 and the Gutt star product on the dual of a Lie algebra in [18]. The hyperbolic disc was treated in [27]. However, the work so far is based on the study of specific examples and a more general approach to these questions has not yet been proposed.

In this work we attempt to lay the foundations to treat the class of coadjoint orbits of (compact semisimple connected) Lie groups in this setting. Coadjoint orbits give examples of a big class of geometrically non-trivial symplectic manifolds, which under certain assumptions on the Lie group 
can be equipped with a complex structure. One of the first interesting examples is the 2 -sphere with its $\mathrm{SO}(3)$-invariant symplectic structure, for which many of the currently available methods in the field fail [36]. Coadjoint orbits have proven useful in many areas of mathematics and physics, e.g. through their relation to unitary representations of Lie groups given by Kirillov's orbit method [24].

The starting point for the present work are the constructions of star products of Wick type due to Karabegov [22,23] and Alekseev-Lachowska [1]. Earlier constructions of star products have been obtained in [8, 9, 12, 15, 29].

Recall the following definition of star products of Wick type [11]: A formal star product on a Kähler manifold $M$ of the form

$$
f * g=\sum_{i=0}^{\infty} z^{i} C_{i}(f, g)
$$

where the $C_{i}$ are bidifferential operators on $M$, is said to be of Wick type if when restricted to any local holomorphic coordinate system, the $C_{i}$ contain only holomorphic derivatives in the first variable and only antiholomorphic derivatives in the second one. Similarly, a star product of anti-Wick type contains only antiholomorphic derivatives in the first variable and holomorphic derivatives in the second one. Both are also known as star products with separation of variables [21]. Note that both Karabegov and Alekseev-Lachowska define products in the non-formal setting for certain functions with rational dependence on $\hbar$. Then they obtain a formal product by considering the pointwise asymptotic expansion around $\hbar=0$. To the best of our knowledge, it only appeared in the literature that the formal expansions agree, see [16, 23], where the Karabegov class is calculated, and this class classifies star products of Wick type uniquely [21]. Our first result, Theorem 3.1 says that the constructions agree on the nose:

Main Theorem I Let a coadjoint orbit of a compact connected semisimple Lie group $K$ with a fixed complex structure be given. Then Karabegov's star product $*_{\hbar}$ for the trivial Karabegov class agrees with the product $*_{\hbar}^{\prime}$ defined by Alekseev-Lachowska on the $K$-finite functions whenever $\hbar$ is different from the countably many poles.

The spirit of the constructions is, however, rather different. Karabegov deforms the Gutt star product [20] on the dual of a Lie algebra in such a way that it becomes tangential to the coadjoint orbits and can actually be restricted. On the other hand Alekseev-Lachowska construct a formula that looks like a Drinfel'd twist. Note also that the construction of Alekseev-Lachowska still works for non-compact semisimple Lie groups as long as the orbit contains a semisimple element. However, we are only interested in the compact case in this paper.

Given a non-formal $K$-invariant Karabegov class with a rational dependence on $\hbar$, we use Karabegov's construction to obtain a star product with rational dependence on $\hbar$ on the polynomials. The formal Karabegov class of this product is the formal expansion of the above chosen class. At the special values of the poles, the product is still defined on a subspace of the polynomials, giving a possibly finite dimensional algebra. As already noticed by Karabegov [23, for the example of the 2 -sphere these algebras are exactly the ones obtained via Berezin-Toeplitz quantization [12 15].

We then restrict to the special case of the 2-sphere and a generic $\hbar \in \mathbb{C}$, meaning that the star product is defined on the whole polynomial algebra. We attack the problem of enlarging these algebras by constructing a locally convex topology in which the product is continuous. Since the algebra of polynomials on the 2-sphere is isomorphic to a quotient of the symmetric algebra over a 3-dimensional vector space, we can look at the quotient topology induced by the $T_{R}$-topology used in e.g. [39], that we call the quotient- $T_{R}$-topology.

Main Theorem II The star product $*_{\hbar}$ on the 2-sphere is continuous with respect to the quotient$T_{R}$-topology for $R \geq 0$ if $\frac{1}{\hbar} \notin \mathbb{N}$. 
Working with the Alekseev-Lachowska construction it is even more natural to consider a topology defined in a slightly different way, that is however equivalent to the quotient- $T_{R}$-topology.

Having found such a topology, we complete the algebra to a Fréchet algebra. Note in particular that the quotient- $T_{R}$-topology is independent of $\hbar$, so the underlying topological vector space of the Fréchet algebras is the same. Surprisingly, the completed algebras are isomorphic to the algebras obtained in [27] for the hyperbolic disc. However, their *-involutions differ. In a follow up paper we will investigate their relations in detail.

In future research, we would also like to extend our convergence results to more coadjoint orbits. The question of convergence relates to the coefficients occurring in the twist-like formula of AlekseevLachowska. These coefficients can be obtained in a purely Lie algebraic framework, so that we hope to answer questions about convergence of the star product by looking at properties of the Lie algebra.

This paper is partially based on the master thesis [37]. It is organized as follows. In Section 2 we recall all the basic notions concerning coadjoint orbits and the construction of complex structures that are needed throughout the paper. Furthermore, we recall the constructions of star products of Wick type on coadjoint orbits of compact semisimple and connected Lie groups obtained by AlekseevLachowska and Karabegov, respectively. Section 3 contains the comparison between the two constructions and we prove Theorem I, Section 4 contains first convergence results for formal star products of Wick-type on coadjoint orbits. Here, we prove that the star product is defined on the subalgebra of polynomials on the orbit. Finally, Section 5 contains the main results of this paper. We discuss the particular case of the 2-sphere interpreted as a coadjoint orbit of $\mathrm{SU}(2)$. We introduce the quotient$T_{R}$-topology and reduction- $T_{R}$-topology and prove that they are equivalent. Furthermore we prove Theorem II

\section{Notation}

We use $\mathscr{C}^{\infty}(M)$ for real valued smooth functions on a manifold $M$ and $\mathscr{C}_{\mathbb{C}}^{\infty}(M)$ for complex valued smooth functions. If $M$ is an immersed submanifold of a vector space $V$, we denote the restriction of complex valued polynomials on $V$ to $M$ by $\operatorname{Pol}(M)$. Moreover, $G$ and $K$ denote Lie groups with Lie algebras $\mathfrak{g}$ and $\mathfrak{k}$, respectively. We always use $z$ to denote a formal parameter, $\hbar$ is a complex number.

\section{Acknowledgements}

We would like thank Matthias Schötz for valuable discussions. The second author is supported by the Danish National Research Foundation through the Centre for Symmetry and Deformation (DNRF92).

\section{Preliminaries}

In this section we collect some known results on the construction of star products on coadjoint orbits which will be used in the sequel. For this purpose, we first recall some basic tools concerning coadjoint orbits which can be found e.g. in [28].

\subsection{Coadjoint orbits and complex structures}

Let $G$ be a real Lie group with Lie algebra $\mathfrak{g}$ and denote by $\Omega_{\xi}$ the coadjoint orbit of $G$ through $\xi \in \mathfrak{g}^{*}$ and by $\mathcal{O}_{\mu}$ the adjoint orbit of $G$ through $\mu \in \mathfrak{g}$. Recall that $\Omega_{\xi}$ has a smooth structure, which makes $\Omega_{\xi}$ an immersed submanifold of $\mathfrak{g}^{*}$ and an embedded submanifold if $G$ is compact. The coadjoint orbits $\Omega_{\xi}$ can be endowed with a $G$-invariant symplectic form $\omega_{\Omega_{\xi}}$, called the Kirillov-Kostant-Souriau (KKS) form.

Lemma 2.1 Let $G$ be a connected semisimple Lie group. Then: 

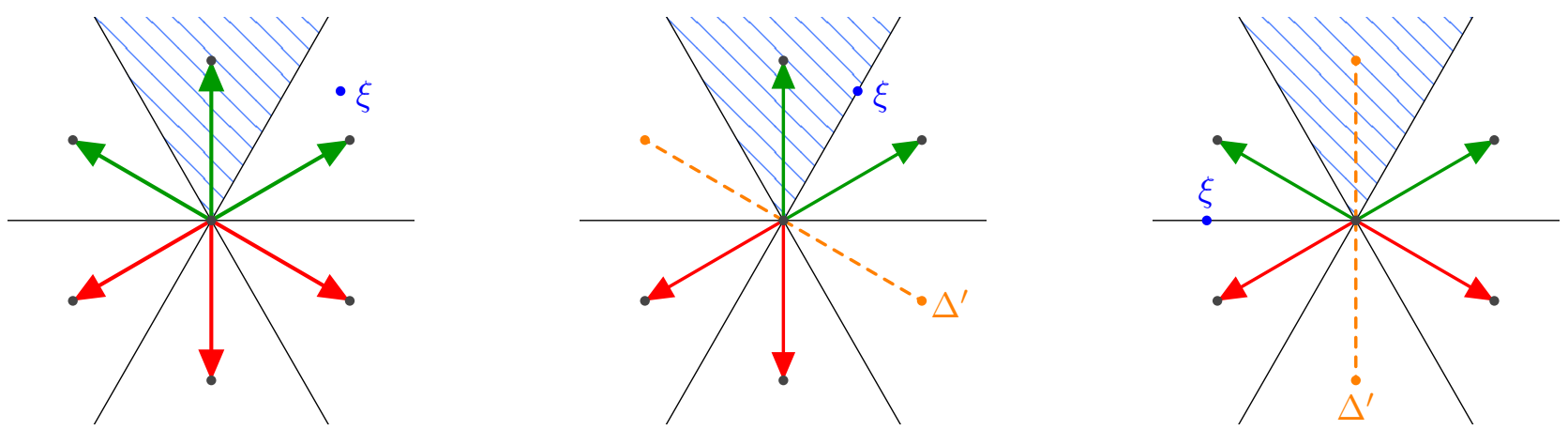

Figure 1: Coadjoint orbits of SU(3). The left example shows a regular orbit of dimension 6 , the other two examples show the same non-regular orbit of dimension 4 which is diffeomorphic to $\mathbb{C P}^{2}$. The figures show $\mathfrak{h}^{*}$ with roots in $\hat{\Delta}^{+}$drawn in green and roots in $\hat{\Delta}^{-}$in red. The ordering in the right example is not invariant, the other two orderings are invariant.

i.) The adjoint and coadjoint orbits are diffeomorphic via the Killing form $B$ of the Lie algebra $\mathfrak{g}$ of $G$.

ii.) Adjoint orbits of $G$ also carry a G-invariant symplectic form $\omega_{\mathcal{O}_{\mu}}$.

In the following we briefly recall the construction of complex structures on adjoint orbits of connected compact semisimple Lie groups (see among others [10] for details, also in the non-compact case), which will be crucial in the construction of star products.

Let us consider a connected, compact and semisimple Lie group $K$ and let $\mathcal{O}_{\mu}$ be its adjoint orbit through $\mu \in \mathfrak{k}$, which is diffeomorphic to the quotient $K / K^{\mu}$ of $K$ by the stabilizer of $\mu$. Let $\mathfrak{k}$ and $\mathfrak{k}^{\mu} \subseteq \mathfrak{k}$ be the Lie algebras of $K$ and $K^{\mu}$, respectively, and denote their complexifications by $\mathfrak{g}$ and $\mathfrak{g}^{\mu}$. Consider the orthogonal complement $\mathfrak{m}$ of $\mathfrak{g}^{\mu}$ under the Killing form $B$, for which we have $\mathfrak{m} \cong \mathfrak{g} / \mathfrak{g}^{\mu}$ as vector spaces. Furthermore, let $\mathfrak{t}$ be a maximally commutative Lie subalgebra of $\mathfrak{k}^{\mu}$ containing $\mu$. It is easy to see that $\mathfrak{t}$ is also maximally commutative in $\mathfrak{k}$, hence its complexification $\mathfrak{h}$ is a Cartan subalgebra of $\mathfrak{g}$ containing $\mu$. Since $\mu$ commutes with $\mathfrak{h}$ and acts as multiplication by $\alpha(\mu)$ on a root space $\mathfrak{g}_{\alpha}$, the subalgebra $\mathfrak{g}^{\mu}$ is a sum of $\mathfrak{h}$ and the root spaces $\mathfrak{g}_{\alpha}$ for which $\alpha(\mu)=0$. Let us denote

$$
\Delta^{\prime}=\{\alpha \in \Delta \mid \alpha(\mu)=0\} \quad \text { and } \quad \hat{\Delta}=\{\alpha \in \Delta \mid \alpha(\mu) \neq 0\} .
$$

In particular, we have

$$
\mathfrak{g}^{\mu}=\mathfrak{h} \oplus \bigoplus_{\alpha \in \Delta^{\prime}} \mathfrak{g}_{\alpha} \quad \text { and } \quad \mathfrak{m}=\bigoplus_{\alpha \in \hat{\Delta}} \mathfrak{g}_{\alpha}
$$

Recall that a subset $\hat{\Delta}^{+} \subseteq \hat{\Delta}$ is said to define an invariant ordering on $\hat{\Delta}$ if for $\hat{\Delta}^{-}:=-\hat{\Delta}^{+}$it satisfies the following identities:

$$
\begin{gathered}
\hat{\Delta}^{+} \cup \hat{\Delta}^{-}=\hat{\Delta} \text { and } \hat{\Delta}^{+} \cap \hat{\Delta}^{-}=\emptyset, \\
\alpha, \beta \in \hat{\Delta}^{+}, \alpha+\beta \in \hat{\Delta} \Longrightarrow \alpha+\beta \in \hat{\Delta}^{+}, \\
\alpha \in \hat{\Delta}^{+}, \gamma \in \Delta^{\prime}, \alpha+\gamma \in \Delta \Longrightarrow \alpha+\gamma \in \hat{\Delta}^{+} .
\end{gathered}
$$

The following theorem can be found e.g. in [10].

Theorem $2.2 K$-invariant complex structures on the adjoint orbit $\mathcal{O}_{\mu}$ are in bijection with invariant orderings on $\hat{\Delta}$. 
In fact, we note that the complexified tangent space $T_{\mu}^{\mathbb{C}} \mathcal{O}_{\mu}$ can be identified with the span of $\mathfrak{g}_{\alpha}$ for $\alpha \in \hat{\Delta}$ via the map

$$
\left\langle\mathfrak{g}_{\alpha} \mid \alpha \in \hat{\Delta}\right\rangle \ni X \longmapsto X_{\mathcal{O}_{\mu}}(\mu):=\left.\frac{\mathrm{d}}{\mathrm{d} t}\right|_{t=0} \operatorname{Ad}_{\exp (t X)} \mu \in T_{\mu}^{\mathbb{C}} \mathcal{O}_{\mu} .
$$

Given an invariant ordering, the corresponding complex structure is given by multiplication by $\mathrm{i}$ on the $\mathfrak{g}_{\alpha}$ with $\alpha \in \hat{\Delta}^{+}$and by multiplication by $-\mathrm{i}$ on $\mathfrak{g}_{-\alpha}$. Invariant orderings always exist as we can choose any linear functional $\ell$ on $\mathfrak{h}^{*}$ that takes real values on all roots, vanishes on $\Delta^{\prime}$ and does not vanish on $\hat{\Delta}$. Then we get an invariant ordering by letting all $\alpha \in \hat{\Delta}^{+}$with $\ell(\alpha)>0$ be positive.

For all $\alpha \in \Delta$ we choose elements $X_{\alpha} \in \mathfrak{g}_{\alpha}, H_{\alpha} \in \mathfrak{h}$ and $Y_{\alpha} \in \mathfrak{g}_{-\alpha}$ satisfying $\left[X_{\alpha}, Y_{\alpha}\right]=H_{\alpha}$, $\left[H_{\alpha}, X_{\alpha}\right]=2 X_{\alpha}$ and $\left[H_{\alpha}, Y_{\alpha}\right]=Y_{\alpha}$ so that they span subalgebras isomorphic to $\mathfrak{s l}_{2}(\mathbb{C})$, as can be seen e.g. in [25, Chapter II.4, (2.26)]. As a consequence of the above theorem we obtain the following proposition.

Proposition 2.3 Let $\mathcal{O}_{\mu}$ be an adjoint orbit of a compact connected semisimple Lie group $K$ and $\lambda:=-\mathrm{i} B(\mu, \cdot) \in \mathfrak{h}^{*}$. Then:

i.) There is a unique $K$-invariant complex structure $J_{\text {Kähler }}$ on $\mathcal{O}_{\mu}$ such that $\mathcal{O}_{\mu}$ endowed with

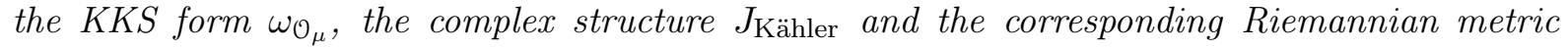
$g(u, v):=\omega_{\mathcal{O}_{\mu}}\left(u, J_{\text {Kähler }} v\right)$ becomes a Kähler manifold.

ii.) The complex structure $J_{\text {Kähler }}$ corresponds to an invariant ordering on $\hat{\Delta}$ for which $\alpha \in \hat{\Delta}$ is positive if and only if $\lambda\left(H_{\alpha}\right)<0$.

\subsection{A construction by Karabegov}

We now recall the construction of star products of Wick type on coadjoint orbits obtained in 23 . (more details on the original construction can also be found in [37]). Notice that, due to the isomorphism between the adjoint and coadjoint orbits stated in Lemma 2.1, we can equivalently apply this construction for adjoint orbits.

Consider a compact connected semisimple Lie group $K$ and an adjoint orbit $\mathcal{O}_{\mu}$, with $\mu \in \mathfrak{k}$. Given a complex $K$-invariant structure $J$ on $\mathcal{O}_{\mu}$ one can construct two commuting complex conjugate representations of the Lie algebra $\mathfrak{k}$ as differential operators on $\mathcal{O}_{\mu}$. These representations can be extended to complex algebra representations of the corresponding complexified universal enveloping algebra $\mathscr{U}(\mathfrak{g})$. Thus, composing those algebra representations with the evaluation at the constant function $\mathbb{1} \in \operatorname{Pol}\left(\mathcal{O}_{\mu}\right)$ we obtain maps

$$
L, R: \mathscr{U}(\mathfrak{g}) \rightarrow \operatorname{Pol}\left(\mathcal{O}_{\mu}\right),
$$

allowing us to push forward the non-commutative product from $\mathscr{U}(\mathfrak{g})$ to $\operatorname{im}(L) \subseteq \operatorname{Pol}\left(\mathcal{O}_{\mu}\right)$, after quotienting out the kernel which turns out to be an ideal. Finally, introducing a parameter $\hbar \in \mathbb{C}$ in the construction of the representations gives rise to a star product of (anti-)Wick type.

In order to construct the representations of $\mathfrak{k}$ mentioned above, one needs to introduce a new class of maps on $\mathcal{O}_{\mu}$.

Definition 2.4 (K-equivariant family) $A K$-equivariant family on $\mathcal{O}_{\mu}$ is defined as a map $\mathfrak{k} \rightarrow$ $\mathscr{C}^{\infty}\left(\mathcal{O}_{\mu}\right), X \mapsto f_{X}$, which is linear and $K$-equivariant with respect to the adjoint action of $K$ on $\mathfrak{k}$ and the shift action on $\mathscr{C}^{\infty}\left(\mathcal{O}_{\mu}\right)$ given by $k \triangleright f(x)=f\left(\operatorname{Ad}_{k^{-1}} x\right)$ for $f \in \mathscr{C}^{\infty}\left(\mathcal{O}_{\mu}\right), k \in K$ and $x \in \mathcal{O}_{\mu}$.

$K$-equivariant families can be characterized as follows.

Lemma $2.5 K$-equivariant families on $\mathcal{O}_{\mu}$ are in one-to-one correspondence with $K$-equivariant maps $\gamma: \mathcal{O}_{\mu} \rightarrow \mathfrak{k}^{*}$ via $f_{X}(x)=\langle\gamma(x), X\rangle$. 
Fixing any $x \in \mathcal{O}_{\mu}$ the map $\gamma \mapsto \gamma(x)$ defines a bijection between $K$-equivariant maps $\gamma: \mathcal{O}_{\mu} \rightarrow \mathfrak{k}^{*}$ and $K^{x}$-invariant elements of $\mathfrak{k}^{*}$. In particular, the space of $K$-equivariant maps $\gamma: \mathcal{O}_{\mu} \rightarrow \mathfrak{k}^{*}$ is a finite dimensional vector space.

Now consider a $K$-invariant complex structure $J$ on $\mathcal{O}_{\mu}$. For $X \in \mathfrak{k}$ we can decompose the fundamental vector field $v_{X}(x):=(-X)_{\mathcal{O}_{\mu}}(x)=\left.\frac{\mathrm{d}}{\mathrm{d} t}\right|_{t=0} \exp (-t X) \triangleright x$ into its $(1,0)$ and $(0,1)$ components with respect to $J$, i.e.

$$
v_{X}=\xi_{X}+\eta_{X} \quad \text { with } \quad \xi_{X} \in \Gamma^{\infty}\left(T^{(1,0)} \mathcal{O}_{\mu}\right) \quad \text { and } \quad \eta_{X} \in \Gamma^{\infty}\left(T^{(0,1)} \mathcal{O}_{\mu}\right)
$$

Note that $v_{X} \in \Gamma^{\infty}(T M) \subseteq \Gamma^{\infty}\left(T^{\mathbb{C}} M\right)$ is real by definition, hence $\overline{\xi_{X}}=\eta_{X}$. Furthermore, since the action of $K$ is holomorphic the vector field $\xi_{X}$ is holomorphic and $\eta_{X}$ is antiholomorphic.

Proposition 2.6 Any $K$-equivariant family $f_{X}$ on $\mathcal{O}_{\mu}$ defines $K$-equivariant commuting complex conjugate representations $\ell, r: \mathfrak{k} \rightarrow \operatorname{DiffOp}\left(\mathcal{O}_{\mu}\right), X \mapsto \ell_{X}:=\ell(X)=\xi_{X}-\mathrm{i} f_{X}$ and $X \mapsto r_{X}:=$ $r(X)=\eta_{X}+\mathrm{i} f_{X}$.

Here, by commuting we mean that $\ell_{X}$ and $r_{Y}$ commute for all $X, Y \in \mathfrak{k}$. For a proof of Proposition 2.6 see 23 .

Given a $K$-equivariant family on $\mathcal{O}_{\mu}$ we extend the representations defined in Proposition 2.6, $X \mapsto \ell_{X}$ and $X \mapsto r_{X}$ of $\mathfrak{k}$ to complex algebra representations of the complexified universal enveloping algebra $\mathscr{U}(\mathfrak{g})$. Let $S$ be the antipode of $\mathscr{U}(\mathfrak{g})$ and $\mathbb{1} \in \mathscr{C}^{\infty}\left(\mathcal{O}_{\mu}\right)$ the constant function.

Lemma 2.7 Let $L(u)=\ell(u) \mathbb{1}$ and $R(u)=r(u) \mathbb{1}$.

i.) We have $L(u)=R(S u)$ for all $u \in \mathscr{U}(\mathfrak{g})$.

ii.) $\operatorname{ker}_{L}$ is a two sided ideal in $\mathscr{U}(\mathfrak{g})$.

Proof: For the first item see [23]. Since $u \mapsto \ell(u)$ is a homomorphism $\operatorname{ker}_{L}$ is a left ideal. Using the first claim and noting that $S$ is an anti-homomorphism concludes the proof.

It is important that the image of $L$ and $R$ is actually contained in $\operatorname{Pol}\left(\mathcal{O}_{\mu}\right) \subseteq \mathscr{C}_{\mathbb{C}}^{\infty}\left(\mathcal{O}_{\mu}\right)$. To see this, we note that the polynomials on $\mathcal{O}_{\mu}$ coincide with the $K$-finite elements of $\mathscr{C}_{\mathbb{C}}^{\infty}\left(\mathcal{O}_{\mu}\right)$, i.e. the smooth functions whose shifts span finite dimensional subspaces, [22, Lemma 15]. Using the definition of a $K$-equivariant family, it is easy to see that all elements of $\operatorname{im}(L)$ and $\operatorname{im}(R)$ are indeed $K$-finite.

Now we introduce a parameter $\hbar$ into the construction. Suppose that the $K$-equivariant family $f_{\bullet}^{(\hbar)}$ on $\mathcal{O}_{\mu}$ depends rationally on $\hbar$ and is regular at $\hbar=0$. In other words, we suppose that

$$
f_{\bullet}^{(\hbar)}=\sum_{j} b_{j}(\hbar) f_{\bullet}^{j}
$$

for a finite number of $K$-equivariant families $f_{\bullet}^{j}$ and rational functions $b_{j}$ regular at $\hbar=0$ (i.e. having no pole at $\hbar=0)$. Denote the set of poles of the functions $b_{j}$ by $P$ and note that it is finite.

Let $\sum_{r \geq 0} z^{r} f_{\bullet, r}$ be the formal expansion of $f_{\bullet}^{(\hbar)}$ around $\hbar=0$. In the following we obtain a formal star product of Wick type from $f_{\bullet}^{(\hbar)}$ and in Theorem 2.12 it is shown that $f_{\bullet}, r$ determines the Karabegov form associated to this star product. In particular $f_{\bullet, 0}$ determines the symplectic form that is deformed.

In order to make sure that $f_{\bullet, r}$ determines the $r$-th order of the Karabegov form, we let $\ell^{(\hbar)}(u)$ with $u \in \mathscr{U}(\mathfrak{g})$ be the operators on $\mathcal{O}_{\mu}$ associated to the $K$-equivariant family $\frac{1}{\hbar} f_{\bullet}^{(\hbar)}$ and let $L^{(\hbar)}(u)=$ $\ell^{(\hbar)}(u) \mathbb{1}$.

Definition 2.8 (The algebra $\left(\mathscr{A}_{\hbar}, *_{\hbar}\right)$ ) For $\hbar \in \mathbb{C} \backslash(P \cup\{0\})$ we define the algebra $\left(\mathscr{A}_{\hbar}, *_{\hbar}\right)$ to be the pushforward of the algebra $\mathscr{U}(\mathfrak{g}) / \operatorname{ker}_{L^{(\hbar)}}$ to $\operatorname{im}\left(L^{(\hbar)}\right) \subseteq \operatorname{Pol}\left(\mathcal{O}_{\mu}\right)$. We let $\left(\mathscr{A}_{0}, *_{0}\right)$ be the polynomial algebra on the orbit with its commutative product. 
From Lemma 2.5 we see that $f_{\bullet}, r$ corresponds to $K$-equivariant maps $\gamma_{r}: \mathcal{O}_{\mu} \rightarrow \mathfrak{k}^{*}$ and the image of each of these maps is a coadjoint orbit $\Omega_{r}$. Denote by $\omega_{\gamma_{r}}$ the pull-back of $\omega_{\Omega_{r}}$ via $\gamma_{r}$. From now on we assume that $f_{\bullet, 0}$ is non-degenerate, meaning that the associated two-form $\omega_{\gamma_{0}}$ is non-degenerate.

Theorem 2.9 (Karabegov [23]) Let $K$ be a connected, compact and semisimple Lie group and $\mathcal{O}_{\mu}$ be its adjoint orbit through $\mu \in \mathfrak{k}$. Fix a K-equivariant family $f_{\bullet}^{(\hbar)}=\sum_{j} b_{j}(\hbar) f_{\bullet}^{j}$, where $b_{j}$ are rational functions of $\hbar$, regular at 0 .

i.) Any $u \in \operatorname{Pol}\left(\mathcal{O}_{\mu}\right)$ can be written as a finite sum

$$
u=\sum_{j} \hbar^{d(j)} a_{j}(\hbar) L^{(\hbar)}\left(u_{j}\right)
$$

with rational functions $a_{j}$, regular at 0 (and depending on $u$ ), and $u_{j} \in \mathscr{U}_{d(j)}(\mathfrak{g})$. Here $\mathscr{U}_{d}(\mathfrak{g})$ denotes the filtration of $\mathscr{U}(\mathfrak{g})$, i.e. $\mathscr{U}_{d}(\mathfrak{g})$ is spanned by products of at most d elements from $\mathfrak{g}$.

ii.) Any function $u \in \operatorname{Pol}\left(\mathcal{O}_{\mu}\right)$ is an element of the algebra $\left(\mathscr{A}_{\hbar}, *_{\hbar}\right)$ for all but a finite number of values of $\hbar$.

iii.) For all but a countable number of values of $\hbar$, the elements of the algebras $\mathscr{A}_{\hbar}$ and $\operatorname{Pol}\left(\mathcal{O}_{\mu}\right)$ coincide.

iv.) If $u, v \in \mathscr{A}_{\hbar}$ and $u$ is written as in (2.10) then

$$
u *_{\hbar} v=\sum_{j} \hbar^{d(j)} a_{j}(\hbar) \ell^{(\hbar)}\left(u_{j}\right) v .
$$

v.) For all $x \in \mathcal{O}_{\mu}, u, v \in \operatorname{Pol}\left(\mathcal{O}_{\mu}\right)$ the function $\hbar \mapsto(u * \hbar v)(x)$ is rational in $\hbar$ with finitely many poles and regular at 0 .

vi.) $*_{\hbar}$ satisfies

$$
\begin{aligned}
u *_{\hbar} v & \rightarrow u v, \\
\hbar^{-1}\left(u *_{\hbar} v-v *_{\hbar} u\right) & \rightarrow \mathrm{i}\{u, v\}
\end{aligned}
$$

for $\hbar \rightarrow 0$ (pointwise), where $\{\cdot, \cdot\}$ is the Poisson bracket corresponding to the symplectic form $\omega_{\gamma_{0}}$.

Note that (2.10) and (2.11) only hold for $\hbar$ different from the set of poles $P$. Let us discuss some properties of $* \hbar$.

Corollary $2.10 \quad$ i.) $*_{\hbar}$ is $K$-invariant, i.e. $k \triangleright\left(u *_{\hbar} v\right)=(k \triangleright u) *_{\hbar}(k \triangleright v)$ for all $k \in K$.

ii.) For every value of $\hbar$ the operator $*_{\hbar}$ is differential on $\mathscr{A}_{\hbar}$ if any of its arguments is fixed.

iii.) For every value of $\hbar$ the star product $*_{\hbar}$ derives the first argument only in antiholomorphic and the second argument only in holomorphic directions with respect to the chosen complex structure $J$.

iv.) $*_{\hbar}$ is of anti-Wick type with respect to the holomorphic structure $J$ on $\mathcal{O}_{\mu}$ (respectively, of Wick type with respect to $J_{\text {Kähler }}$ if we choose $J$ opposite to $\left.J_{\text {Kähler }}\right)$.

v.) For every value of $\hbar$ and $u \in \mathscr{A}_{\hbar}$ we have $\bar{u} \in \mathscr{A}_{\bar{\hbar}}$. In particular for $\hbar \in \mathbb{R} \backslash P, \mathscr{A}_{\hbar}$ is closed under complex conjugation.

vi.) For every value of $\hbar$ the star product $*_{\hbar}$ is Hermitian, meaning that $\overline{u *_{\hbar} v}=\bar{v} *_{\bar{\hbar}} \bar{u}$.

Note that we cannot expect $*_{\hbar}$ to be a bidifferential operator since it will usually contain derivatives of arbitrary high order if no argument is fixed. Let us consider the asymptotic expansion of the star product, which can be obtained from (2.11). Since by Theorem 2.9v.) $u * \hbar v(x)$ is a rational function 
of $\hbar$ with no pole at zero it has an absolutely and uniformly convergent Taylor series in a small enough neighbourhood of $\hbar=0$. We set

$$
u \star v=\left.\sum_{r=0}^{\infty} \frac{1}{r !} z^{r} \frac{\mathrm{d}^{r}}{\mathrm{~d} \hbar^{r}}\right|_{\hbar=0} u * \hbar v
$$

for $u, v \in \operatorname{Pol}\left(\mathcal{O}_{\mu}\right)$. From (2.11) we get that in order $r$ for all $u \in \operatorname{Pol}\left(\mathcal{O}_{\mu}\right), u \star$. is a differential operator of order at most $r$ and $\cdot \star u$ is also a differential operator of order at most $r$.

As a direct consequence of the previous corollary we can prove the following statement.

Corollary 2.11 The asymptotic expansion $\star$ of $*_{\hbar}$ is a $K$-invariant, differential, natural, Hermitian formal star product of anti-Wick type with respect to $J$.

Formal star products of Wick type on a symplectic manifold $\left(M, \omega_{0}\right)$ are classified by formal closed $(1,1)$-forms, see [21,34]. Indeed for any formal closed $(1,1)$-form $\omega=\sum_{r=0}^{\infty} z^{r} \omega_{r}$, there is a unique star product of Wick type on $\left(M, \omega_{0}\right)$ such that for all $f \in \mathscr{C}_{\mathbb{C}}^{\infty}(M),\left.\left.f\right|_{U} \star\right|_{U} \cdot$ commutes with all holomorphic functions and the operators $\frac{\partial \Phi}{\partial z^{k}}+\nu \frac{\partial}{\partial z^{k}}$ and such that $\left.\left.\cdot \star\right|_{U} f\right|_{U}$ commutes with all antiholomorphic functions and the operators $\frac{\partial \Phi}{\partial \bar{z}^{k}}+\nu \frac{\partial}{\partial \bar{z}^{k}}$. Here $U$ is any holomorphic chart with local coordinates $z^{1}, \ldots, z^{n}$ and $\Phi$ is a potential for $\omega$ on $U$. Vice versa, the star product determines $\omega$ with these properties uniquely.

Theorem 2.12 (Karabegov [23]) Let $\mathcal{O}_{\mu}$ be an adjoint orbit of a compact connected semisimple

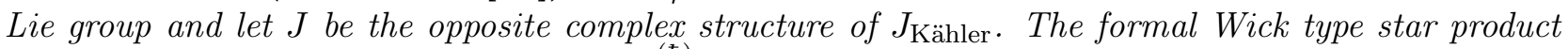

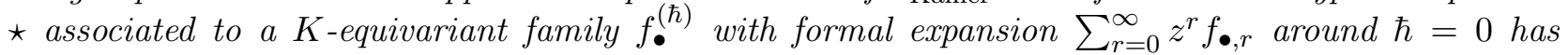
Karabegov form $\omega=\sum_{r=0}^{\infty} z^{r} \omega_{\gamma_{r}}$, where $\omega_{\gamma_{r}}$ is the pullback of the KKS symplectic form from $\gamma_{r}\left(\mathcal{O}_{\mu}\right)$ to $\mathcal{O}_{\mu}$ via the map $\gamma_{r}$ corresponding to $f_{\bullet}, r$. In particular it deforms the symplectic manifold $\left(\mathcal{O}_{\mu}, \omega_{\gamma_{0}}\right)$.

Note that $\omega_{\gamma_{0}}$ coincides with the KKS symplectic form if we choose $f_{\bullet}^{(\hbar)}$ such that $f_{X, 0}(x)=B(x, X)$ holds for all $X \in \mathfrak{k}$ and $x \in \mathcal{O}_{\mu} \subseteq \mathfrak{k}$.

\subsection{A construction by Alekseev-Lachowska}

The second construction that we recall is due to Alekseev and Lachowska [1]. The main idea is to use a certain pairing defined below, that is associated to a decomposition of the Lie algebra, to define an operator depending rationally on $\hbar$. Its asymptotic expansion gives again an invariant star product of Wick type. We want to remark here that the construction really builds on the splitting described below and this splitting is available also in some nilpotent or infinite dimensional examples, as well as for adjoint orbits of non-compact semisimple Lie groups that contain a semisimple element.

Recall that taking left invariant vector fields $X \mapsto X^{\text {left }}$ extends to an isomorphism between the universal enveloping algebra $\mathscr{U}\left(\mathfrak{g}_{\mathbb{C}}\right)$ of the complexification $\mathfrak{g}_{\mathbb{C}}$ of $\mathfrak{g}$ and the space of $G$-invariant differential operators $\operatorname{DiffOp}^{G}(G)$ on a Lie group $G$.

We are interested in the space of $G$-invariant differential operators on $G / H$, where $H$ is a closed subgroup of $G$. Any $f \in \mathscr{C}_{\mathbb{C}}^{\infty}(G / H)$ can be extended to a function $\tilde{f} \in \mathscr{C}_{\mathbb{C}}^{\infty}(G)$ that is invariant under right shifts by $H$. The function $X_{1}^{\text {left }} \cdots X_{n}^{\text {left }} \tilde{f}$ is right $H$-invariant again if and only if $X_{1} \cdots X_{n} \in \mathscr{U}\left(\mathfrak{g}_{\mathbb{C}}\right)$ is invariant under the adjoint action of $H$. Since $X^{\text {left }}$ kills $\tilde{f}$ if $X \in \mathfrak{h}$, this can be used to construct an isomorphism between $\left(\mathscr{U}\left(\mathfrak{g}_{\mathbb{C}}\right) /\left(\mathscr{U}\left(\mathfrak{g}_{\mathbb{C}}\right) \cdot \mathfrak{h}_{\mathbb{C}}\right)\right)^{H}$ and the space of $G$-invariant differential operators $\operatorname{DiffOp}^{G}(G / H)$ on a homogeneous space $G / H$. By the superscript $H$ we mean the elements invariant under the adjoint action of $H$. Note that the adjoint action of $H$ on $\mathscr{U}\left(\mathfrak{g}_{\mathbb{C}}\right)$ fixes the left ideal $\mathscr{U}\left(\mathfrak{g}_{\mathbb{C}}\right) \cdot \mathfrak{h}_{\mathbb{C}}$ and is therefore well-defined on the quotient. 

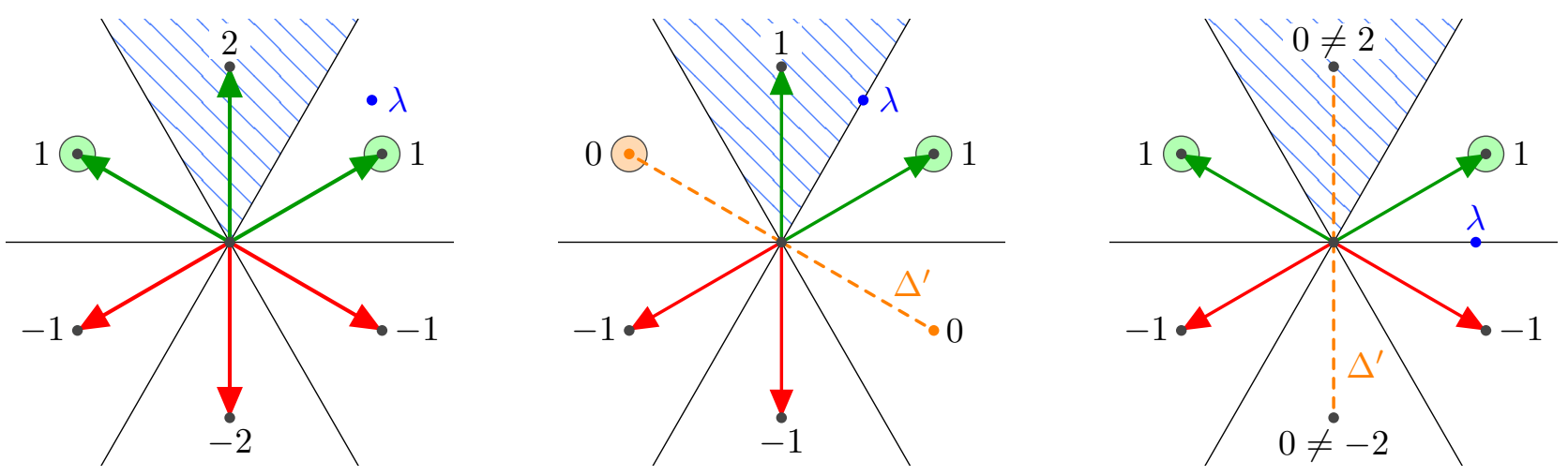

Figure 2: Illustration of the grading. Fundamental roots are encircled. Positive roots are drawn green and negative ones red if they lie in $\hat{\Delta}$. Roots from $\Delta^{\prime}$ are drawn with orange dashed lines. The grading is indicated next to each root space. The Cartan subalgebra has always grading 0 . A regular orbit of $\mathrm{SU}(3)$ is shown on the left, the other two pictures are of non-regular orbits, $\lambda=B(-\mathrm{i} \mu, \cdot)$. In the right picture the ordering on $\Delta^{\prime}$ is not invariant and therefore the grading is not well-defined.

A formal $G$-invariant star product on $G / H$ is defined by a series of $G$-invariant bidifferential operators on $G / H$, i.e. an element

$$
F \in\left(\left(\mathscr{U}\left(\mathfrak{g}_{\mathbb{C}}\right) /\left(\mathscr{U}\left(\mathfrak{g}_{\mathbb{C}}\right) \cdot \mathfrak{h}_{\mathbb{C}}\right)\right)^{\otimes 2}\right)^{H}[[z]]
$$

satisfying some further properties that assure associativity and the usual requirements for zeroth and first order terms. Here the $H$-action is the diagonal action of $H$ on the tensor product.

We only treat the case of adjoint orbits of compact semisimple connected Lie groups here and set our notation accordingly. Note however, that the following would still make sense for any grading of $\mathfrak{g}$ preserved under the adjoint action of $H$.

From now on we let $G:=K$ be a compact connected semisimple Lie group and $H:=K^{\mu}$ the stabilizer of some $\mu \in \mathfrak{k}$, where $\mathfrak{k}$ and $\mathfrak{k}^{\mu}$ denote the Lie algebras of $K$ and $K^{\mu}$, respectively. Denote their complexifications by $\mathfrak{g}$ and $\mathfrak{g}^{\mu}$. Choose a Cartan subalgebra $\mathfrak{h}$ of $\mathfrak{g}$ containing $\mu$, define roots $\Delta$ and subsets $\Delta^{\prime}, \hat{\Delta}$ as in (2.1) and choose an ordering on $\Delta$ such that the induced ordering on $\hat{\Delta}$ is invariant. Let $J$ be the corresponding complex structure. Define a $\mathbb{Z}$-grading of $\mathfrak{g}$ (the graded components being called $\mathfrak{g}_{n}$ ) in the following way: firstly, let $\mathfrak{g}_{0}=\mathfrak{g}^{\mu}$ and secondly, let all other elements of the root spaces corresponding to fundamental roots that are not already in $\mathfrak{g}_{0}$ have degree 1, i.e. $\mathfrak{g}_{1}=\bigoplus_{\alpha \in \Sigma \backslash \Delta^{\prime}} \mathfrak{g}_{\alpha}$. This gives a well-defined grading since the ordering of $\hat{\Delta}$ is invariant.

Define $\mathfrak{n}^{+}=\oplus_{i \geq 1} \mathfrak{g}_{i}$ and $\mathfrak{n}^{-}=\oplus_{i \leq-1} \mathfrak{g}_{i}$ and denote the projection to $\mathfrak{g}_{0}$ in the direct sum $\mathfrak{g}=$ $\mathfrak{g}_{0} \oplus \mathfrak{n}^{+} \oplus \mathfrak{n}^{-}$by pr. Let $\lambda: \mathfrak{g}_{0} \rightarrow \mathbb{C}$ be a Lie algebra homomorphism such that the pairing

$$
\mathfrak{n}^{+} \times \mathfrak{n}^{-} \rightarrow \mathbb{C}: \quad(u, v) \mapsto \lambda(\operatorname{pr}([u, v]))
$$

is non-degenerate. We are mainly interested in the case $\lambda=B(-\mathrm{i} \mu, \cdot)$. Depending on the context we extend $\lambda$ by zero on $\mathfrak{n}^{+}$and $\mathfrak{n}^{-}$, still denoted by $\lambda$. For any $\hbar \in \mathbb{C} \backslash\{0\}$ we consider the pairing (which is related to the Shapovalov pairing of the corresponding Verma modules)

$$
(\cdot, \cdot)_{\hbar}: \mathscr{U}\left(\mathfrak{n}^{-}\right) \times \mathscr{U}\left(\mathfrak{n}^{+}\right) \rightarrow \mathbb{C}, \quad(y, x) \mapsto(y, x)_{\hbar}=\left\langle\frac{\lambda}{\hbar},(S(x) y)_{0}\right\rangle .
$$

Here $(\cdot)_{0}$ is the projection onto the second summand in $\mathscr{U}(\mathfrak{g})=\left(\mathfrak{n}^{-} \mathscr{U}(\mathfrak{g})+\mathscr{U}(\mathfrak{g}) \mathfrak{n}^{+}\right) \oplus \mathscr{U}\left(\mathfrak{g}_{0}\right)$.

It is important to notice that $\mathscr{U}(\mathfrak{g})$ is graded: we can simply define the grade of an element $X_{1} \cdots X_{n} \in \mathscr{U}(\mathfrak{g})$ to be the sum of the grades of the $X_{i} \in \mathfrak{g}$. This is well defined, since the ideal generated by $X Y-Y X-[X, Y]$ is homogeneous. The graded components of $\mathscr{U}\left(\mathfrak{n}^{-}\right)$and $\mathscr{U}\left(\mathfrak{n}^{+}\right)$are 
all finite dimensional and the pairing in (2.17) respects the grading in the sense that for homogeneous elements $x \in \mathscr{U}\left(\mathfrak{n}^{-}\right)$and $y \in \mathscr{U}\left(\mathfrak{n}^{+}\right)$it is non-zero only if the degrees of $x$ and $y$ add up to zero. Thus we can choose homogeneous bases of $\mathscr{U}\left(\mathfrak{n}^{-}\right)$and $\mathscr{U}\left(\mathfrak{n}^{+}\right)$such that the pairing is block diagonal with each block being finite dimensional. With a careful analysis of the pairing one can then prove that each block is invertible with only finitely many exceptions for $\hbar$, see [1, Proposition 3.1].

Proposition 2.13 The pairing $(\cdot, \cdot)_{\hbar}$ is non-singular for almost all $\hbar \in \mathbb{C} \backslash\{0\}$.

Here by non-singular we mean that for all $x \in \mathscr{U}\left(\mathfrak{n}^{-}\right)$there is $y \in \mathscr{U}\left(\mathfrak{n}^{+}\right)$with $(x, y)_{\hbar} \neq 0$. Consequently we can choose bases $\left\{1, y_{1}^{\hbar}, y_{2}^{\hbar}, \ldots\right\}$ of $\mathscr{U}\left(\mathfrak{n}^{-}\right)$and $\left\{1, x_{1}^{\hbar}, x_{2}^{\hbar}, \ldots\right\}$ of $\mathscr{U}\left(\mathfrak{n}^{+}\right)$ordered by increasing grading for almost all $\hbar \in \mathbb{C}$, that are dual to each other with respect to $(\cdot, \cdot)_{\hbar}$. Then

$$
F_{\hbar}=1 \otimes 1+y_{1}^{\hbar} \otimes x_{1}^{\hbar}+y_{2}^{\hbar} \otimes x_{2}^{\hbar}+\cdots \in \mathscr{U}\left(\mathfrak{n}^{-}\right) \hat{\otimes} \mathscr{U}\left(\mathfrak{n}^{+}\right)
$$

is well-defined, independent of the bases chosen. Note that of the infinitely many terms appearing in the formula for $F_{\hbar}$ only finitely many lie in a certain grade. Finally, in [1, Theorem 4.9] the authors prove the following result.

Theorem 2.14 (Alekseev-Lachowska) Let $\mathcal{O}_{\mu}$ be an adjoint orbit of a compact connected semisimple Lie group $K$. With the notation introduced above

i.) $F_{\hbar}$ depends rationally on $\hbar$, with no pole at zero.

ii.) The formal Taylor series expansion of $F_{\hbar}$ around 0 gives an element $F \in((\mathscr{U}(\mathfrak{g}) /(\mathscr{U}(\mathfrak{g})$. $\left.\left.\left.\mathfrak{g}^{\mu}\right)\right)^{\otimes 2}\right)^{K^{\mu}}[[z]]$. The elements $F_{\hbar}$ and $F$ satisfy an associativity condition.

Remark 2.15 Part i.) of the previous theorem has to be understood in the sense that up to a certain degree in $\mathscr{U}\left(\mathfrak{n}^{-}\right) \hat{\otimes} \mathscr{U}\left(\mathfrak{n}^{+}\right)$the element $F_{\hbar}$ is meromorphic. A priori we cannot say that the poles up to arbitrary degree behave nicely, meaning that we cannot exclude the case that the (countable) set of poles has accumulation points different from zero. However, in the example of the 2-sphere the explicit calculations in later sections show that this is not the case, i.e. for the two sphere 0 is the only accumulation point.

Note that the asymptotic expansion is well-defined because only finitely many terms of $F_{\hbar}$ contribute to a certain degree, due to the fact that the asymptotic expansion of $y_{n}^{\hbar} \otimes x_{n}^{\hbar}$ will have increasing powers of $\hbar$ as $n \rightarrow \infty$. See [1, Remark 3.4] for details.

The next subsection will show that only finitely many terms in (2.18) contribute when applying $F_{\hbar}$ to two polynomials $p, q \in \operatorname{Pol}\left(K / K^{\mu}\right)$. Thus, the corresponding star product

$$
p *_{\hbar}^{\prime} q:=F_{\hbar}(p, q)
$$

is well-defined for $\hbar$ different from the poles. If $\lambda=B(-\mathrm{i} \mu, \cdot)$, the formal star product deforms the the Kirillov-Kostant-Souriau Poisson structure for adjoint orbits of connected semisimple compact Lie groups.

\section{The products of Karabegov and Alekseev-Lachowska agree}

We here show that the product by Alekseev-Lachowska from Theorem 2.14 coincides with the star product of Karabegov defined in Theorem 2.9. Note that $\lambda: \mathfrak{g}_{0} \rightarrow \mathbb{C}$ in the following theorem can be any Lie algebra homomorphism with $\lambda\left(\mathfrak{g}_{0} \cap \mathfrak{k}\right) \subseteq \mathrm{i} \mathbb{R}$, but only for the special choice $\lambda=B(-\mathrm{i} \mu, \cdot)$ does the star product deform the KKS symplectic form. 
Theorem 3.1 Let $\mathcal{O}_{\mu}$ be a fixed adjoint orbit of a compact connected semisimple Lie group with a fixed complex structure $J$. Then Karabegov's star product $*_{\hbar}$ with respect to the $K$-equivariant family defined by $f_{X}^{(\hbar)}(\mu)=\mathrm{i} \lambda(X)$ agrees with the product $*_{\hbar}^{\prime}$ defined by Alekseev and Lachowska with respect to $\lambda$ whenever $\hbar$ is different from the countably many poles.

In order to prove Theorem 3.1 we introduce a function $s_{\lambda}$, already defined in [22] by

$$
s_{\lambda}: \mathscr{U}(\mathfrak{g}) \rightarrow \mathscr{C}_{\mathbb{C}}^{\infty}(K), \quad s_{\lambda} u(k)=\left\langle\lambda,\left(\operatorname{Ad}_{k^{-1}} u\right)_{0}\right\rangle .
$$

Furthermore we let

$$
\psi_{\mu}: K \rightarrow K / K^{\mu} \cong \mathcal{O}_{\mu}
$$

be the projection. It is clear that both $s_{\lambda}$ and $\psi_{\mu}$ are $K$-equivariant, where $K$ acts under the adjoint action on $\mathscr{U}(\mathfrak{g})$ and by left translations on $\mathscr{C}_{\mathbb{C}}^{\infty}(K)$.

Lemma 3.2 Taking the $K$-equivariant family $f_{X}^{(\hbar)}: \mathcal{O}_{\mu} \rightarrow \mathbb{R}$ defined by $f_{X}^{(\hbar)}(\mu)=\mathrm{i} \lambda(X)$, we have

$$
L^{(\hbar)}(u) \circ \psi_{\mu}=s_{\lambda / \hbar} u
$$

for all $u \in \mathscr{U}(\mathfrak{g})$.

Proof: By $K$-equivariance of all involved maps, it suffices to check this at the unit $e \in K$. Hence we need to see that $L^{(\hbar)}(u)(\mu)=\left\langle\lambda / \hbar, u_{0}\right\rangle$ holds. First, if $X=E-\mathrm{i} J E \in \mathfrak{n}^{+}$with $E \in \mathfrak{k} \cap \bigcup_{\alpha \in \hat{\Delta}} \mathfrak{g}_{\alpha}$ then

$$
r^{(\hbar)}(X)=\left(-X_{\mathcal{O}_{\mu}}\right)^{(0,1)}+\frac{\mathrm{i}}{\hbar} f_{X}^{(\hbar)},
$$

and both $-X_{\mathcal{O}_{\mu}}^{(0,1)}$ and $f_{X}^{(\hbar)}$ vanish at $\mu$. Similarly, if $Y \in \mathfrak{n}^{-}$then $\ell^{(\hbar)}(Y)=\left(-Y_{\mathcal{O}_{\mu}}\right)^{(1,0)}-\frac{\mathrm{i}}{\hbar} f_{Y}^{(\hbar)}$ vanishes at $\mu$. It suffices to prove the statement for $u$ in canonical form, i.e. $u=Y_{1} \cdots Y_{n} H_{1} \cdots H_{m} X_{1} \cdots X_{k}$ with $Y_{i} \in \mathfrak{n}^{-}, X_{i} \in \mathfrak{n}^{+}$and $H_{i} \in \mathfrak{g}_{0}$. If $n \geq 1$ then

$$
L^{(\hbar)}(u)(\mu)=\ell^{(\hbar)}\left(Y_{1}\right) \ell^{(\hbar)}\left(Y_{2} \cdots Y_{n} H_{1} \cdots H_{m} X_{1} \cdots X_{k}\right) \mathbb{1}(\mu)=0=\left\langle\frac{\lambda}{\hbar}, u_{0}\right\rangle
$$

and similarly if $k \geq 1$

$$
\begin{aligned}
L^{(\hbar)}(u)(\mu) & =R^{(\hbar)}(S(u))(\mu) \\
& =r^{(\hbar)}\left(-X_{k}\right) r^{(\hbar)}\left(S\left(Y_{1} \cdots Y_{n} H_{1} \cdots H_{m} X_{1} \cdots X_{k-1}\right)\right) \mathbb{1}(\mu) \\
& =0 \\
& =\left\langle\frac{\lambda}{\hbar}, u_{0}\right\rangle .
\end{aligned}
$$

Finally, for $n=k=0$ we have $v_{H}(\mu)=0$ for $H \in \mathfrak{g}_{0}$ and therefore $\xi_{H}(\mu)=\eta_{H}(\mu)=0$. So

$$
\begin{aligned}
L^{(\hbar)}(u)(\mu) & =\frac{-\mathrm{i}}{\hbar} f_{H_{1}}^{(\hbar)}(\mu) \cdots \frac{-\mathrm{i}}{\hbar} f_{H_{m}}^{(\hbar)}(\mu) \\
& =\left(\frac{-\mathrm{i}}{\hbar}\right)^{m} \mathrm{i} \lambda\left(H_{1}\right) \cdots \mathrm{i} \lambda\left(H_{m}\right) \\
& =\frac{1}{\hbar^{m}} \lambda\left(H_{1}\right) \cdots \lambda\left(H_{m}\right) \\
& =\left\langle\frac{\lambda}{\hbar}, u_{0}\right\rangle .
\end{aligned}
$$


In the following for $X=E+\mathrm{i} F \in \mathfrak{g}$ with $E, F \in \mathfrak{k}$ let $\left.X_{M}\right|_{x}:=\left.E_{M}\right|_{x}+\left.\mathrm{i} F_{M}\right|_{x} \in T_{x}^{\mathbb{C}} M$, where $M$ is a manifold with an action of $K$, e.g. $K$ or $\mathcal{O}_{\mu}$.

We enumerate the positive roots by $\alpha_{1}, \ldots, \alpha_{N}$ and let $A \in\{1, \ldots, N\}^{L}$ be a tupel of $L$ elements. Let $X_{\alpha_{i}}, H_{\alpha_{i}}, Y_{\alpha_{i}}$ span subalgebras of $\mathfrak{g}$ isomorphic to $\mathfrak{s l}_{2}$ as in Subsection 2.1.

Lemma 3.3 We have

$$
\mathscr{L}_{X_{A} \text { left }} s_{\lambda} u(e)=s_{\lambda}\left(S\left(X_{A}\right) u\right)(e) \quad \text { and } \quad \mathscr{L}_{Y_{A} \text { left }} s_{\lambda} u(e)=s_{\lambda}\left(u Y_{A}\right)(e),
$$

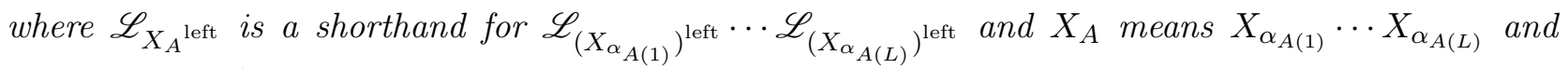
similarly for $\mathscr{L}_{Y_{A} \text { left }}$ and $Y_{A}$.

Proof: Left invariant vector fields are the fundamental vector fields of the right action of $K$ on $K$ by right multiplications. Since there exists a complex Lie group $G$ corresponding to $\mathfrak{g}$ and all involved maps are complex linear, we can also obtain the fundamental vector field of $X \in \mathfrak{g}$ by taking the fundamental vector field of the right action of $G$ on $G$. Hence

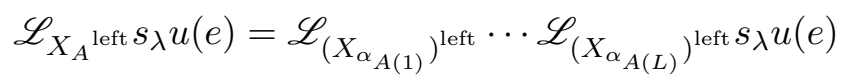

$$
\begin{aligned}
& =\left.\left.\frac{\mathrm{d}}{\mathrm{d} t_{1}}\right|_{t_{1}=0} \cdots \frac{\mathrm{d}}{\mathrm{d} t_{L}}\right|_{t_{L}=0} s_{\lambda} u\left(\exp \left(t_{1} X_{\alpha_{A(1)}}\right) \cdots \exp \left(t_{L} X_{\alpha_{A(L)}}\right)\right) \\
& =\left.\left.\frac{\mathrm{d}}{\mathrm{d} t_{1}}\right|_{t_{1}=0} \cdots \frac{\mathrm{d}}{\mathrm{d} t_{L}}\right|_{t_{L}=0}\left\langle\lambda,\left(\operatorname{Ad}_{\left.\left.\left(\exp \left(t_{1} X_{\alpha_{A(1)}}\right) \cdots \exp \left(t_{L} X_{\alpha_{A(L)}}\right)\right)^{-1} u\right)_{0}\right\rangle}\right\rangle\right. \\
& =\left.\left.\frac{\mathrm{d}}{\mathrm{d} t_{1}}\right|_{t_{1}=0} \cdots \frac{\mathrm{d}}{\mathrm{d} t_{L}}\right|_{t_{L}=0}\left\langle\lambda,\left(\operatorname{Ad}_{\exp \left(-t_{L} X_{\alpha_{A(L)}}\right)} \cdots \operatorname{Ad}_{\exp \left(-t_{1} X_{\alpha_{A(1)}}\right)} u\right)_{0}\right\rangle \\
& =\left\langle\lambda,\left(\left.\left.\frac{\mathrm{d}}{\mathrm{d} t_{L}}\right|_{t_{L}=0} \exp \left(-t_{L} \operatorname{ad}_{X_{\alpha_{A(L)}}}\right) \cdots \frac{\mathrm{d}}{\mathrm{d} t_{1}}\right|_{t_{1}=0} \exp \left(-t_{1} \operatorname{ad}_{X_{\alpha_{A(1)}}}\right) u\right)_{0}\right\rangle \\
& =\left\langle\lambda,\left(\left(-\operatorname{ad}_{X_{\alpha_{A(L)}}}\right) \cdots\left(-\operatorname{ad}_{X_{\alpha_{A(1)}}}\right) u\right)_{0}\right\rangle \\
& =\left\langle\lambda,\left(\left(-X_{\alpha_{A(L)}}\right) \cdots\left(-X_{\alpha_{A(1)}}\right) u\right)_{0}\right\rangle \\
& =\left\langle\lambda,\left(S\left(X_{\alpha_{A(1)}} \cdots X_{\alpha_{A(L)}}\right) u\right)_{0}\right\rangle \\
& =\left\langle\lambda,\left(S\left(X_{A}\right) u\right)_{0}\right\rangle \\
& =s_{\lambda}\left(S\left(X_{A}\right) u\right)(e) \text {. }
\end{aligned}
$$

Here we used that $\left(u X_{\alpha_{i}}\right)_{0}$ vanishes for all $1 \leq i \leq N$ and all $u \in \mathscr{U}(\mathfrak{g})$. The calculation for $Y$ is similar except that now $\left(Y_{\alpha_{i}} u\right)_{0}$ vanishes and therefore

$$
\left\langle\lambda,\left(\left(-\operatorname{ad}_{Y_{\alpha_{A}(L)}}\right) \cdots\left(-\operatorname{ad}_{Y_{\alpha_{A(1)}}}\right) u\right)_{0}\right\rangle=\left\langle\lambda,\left(u Y_{\alpha_{A(1)}} \cdots Y_{\alpha_{A(L)}}\right)_{0}\right\rangle=s_{\lambda}\left(u Y_{A}\right)(e) .
$$

Finally, we can complete the comparison of the star products defined by Karabegov and AlekseevLachowska.

Proof of Theorem 3.1; Fix an adjoint orbit $\mathcal{O}_{\mu}$. Since both star products are $K$-invariant it suffices to check that they agree at $\mu$. Fix two functions $u, v \in \operatorname{Pol}\left(\mathcal{O}_{\mu}\right)$. By Theorem 2.9, , .) we have

$$
u=\sum_{i=1}^{m} \hbar^{d(i)} a_{i}(\hbar) L^{(\hbar)}\left(u_{i}\right) \quad \text { and } \quad v=\sum_{j=1}^{\ell} \hbar^{e(j)} b_{j}(\hbar) L^{(\hbar)}\left(v_{j}\right),
$$

where $a_{i}, b_{j}$ are rational functions of $\hbar$, regular at 0 and $u_{i} \in \mathscr{U}_{d(i)}(\mathfrak{g}), v_{j} \in \mathscr{U}_{e(j)}(\mathfrak{g})$. Consequently

$$
u \circ \psi_{\mu}=\sum_{i=1}^{m} \hbar^{d(i)} a_{i}(\hbar) s_{\lambda / \hbar} u_{i}, \quad v \circ \psi_{\mu}=\sum_{j=1}^{\ell} \hbar^{e(j)} b_{j}(\hbar) s_{\lambda / \hbar} v_{j}
$$


and

$$
\begin{aligned}
\left(u *_{\hbar} v\right) \circ \psi_{\mu} & =\sum_{i=1}^{m} \sum_{j=1}^{\ell} \hbar^{d(i)+e(j)} a_{i}(\hbar) b_{j}(\hbar) L^{(\hbar)}\left(u_{i} v_{j}\right) \circ \psi_{\lambda} \\
& =\sum_{i=1}^{m} \sum_{j=1}^{\ell} \hbar^{d(i)+e(j)} a_{i}(\hbar) b_{j}(\hbar) s_{\lambda / \hbar}\left(u_{i} v_{j}\right)
\end{aligned}
$$

by Lemma 3.2, Let $\left\{y_{0}^{\hbar}:=1, y_{1}^{\hbar}, y_{2}^{\hbar}, \ldots\right\}$ and $\left\{x_{0}^{\hbar}:=1, x_{1}^{\hbar}, x_{2}^{\hbar}, \ldots\right\}$ be the bases of $\mathscr{U}\left(\mathfrak{n}^{-}\right)$and $\mathscr{U}\left(\mathfrak{n}^{+}\right)$used in Subsection 2.3 to construct $F_{\hbar}$. Enumerate the positive roots as above and write $y_{n}^{\hbar}=\sum_{p=1}^{m_{n}} c_{n, p}^{\hbar} Y_{N_{n, p}}$ for complex coefficients $c_{n, p}^{\hbar}$ and tuples $N_{n, p} \in\{1, \ldots, N\}^{L_{n, p}}$ of length $L_{n, p}$. Similarly, write $x_{n}^{\hbar}=\sum_{q=1}^{\ell_{n}} d_{n, q}^{\hbar} X_{M_{n, q}}$. We drop all sums over $p$ and $q$ below. Then

$$
\begin{aligned}
& (u * \hbar v)(\mu) \stackrel{(1)}{=} \sum_{i, j=1}^{m, \ell} a_{i}(\hbar) b_{j}(\hbar) \hbar^{d(i)+e(j)} s_{\lambda / \hbar}\left(u_{i} v_{j}\right)(e) \\
& \stackrel{(2)}{=} \sum_{i, j=1}^{m, \ell} \sum_{n=0}^{\infty} a_{i}(\hbar) b_{j}(\hbar) \hbar^{d(i)+e(j)} s_{\lambda / \hbar}\left(u_{i} c_{n, p}^{\hbar} Y_{N_{n, p}}\right)(e) s_{\lambda / \hbar}\left(S\left(d_{n, q}^{\hbar} X_{M_{n, q}}\right) v_{j}\right)(e) \\
& \stackrel{(3)}{=} \sum_{i, j=1}^{m, \ell} \sum_{n=0}^{\infty} a_{i}(\hbar) b_{j}(\hbar) \hbar^{d(i)+e(j)} c_{n, p}^{\hbar} \mathscr{L}_{\left(Y_{N_{n}, p}\right)}{ }^{\text {left } s_{\lambda / \hbar}}\left(u_{i}\right)(e) d_{n, q}^{\hbar} \mathscr{L}_{\left(X_{M_{n}, q}\right)}{ }^{\text {left }} s_{\lambda / \hbar}\left(v_{j}\right)(e) \\
& \left.\stackrel{(4)}{=} \sum_{n=0}^{\infty} c_{n, p}^{\hbar} \mathscr{L}_{\left(Y_{N_{n, p}}\right)} \text { left }\left(u \circ \psi_{\lambda}\right)(e) d_{n, q}^{\hbar} \mathscr{L}_{\left(X_{M_{n, q}}\right)}\right)^{\text {left }}\left(v \circ \psi_{\lambda}\right)(e) \\
& \stackrel{(5)}{=} F_{\hbar}(u, v)(\mu)=\left(u *_{\hbar}^{\prime} v\right)(\mu) \text {. }
\end{aligned}
$$

Here (1) is (3.6), (2) holds because $\left\{y_{0}:=1, y_{1}^{\hbar}, y_{2}^{\hbar}, \ldots\right\}$ and $\left\{x_{0}:=1, x_{1}^{\hbar}, x_{2}^{\hbar}, \ldots\right\}$ form dual bases with respect to the pairing (2.17). Note that only finitely many terms contribute to the sum over $n$, so there are no convergence issues. The argument in (3) is simply Lemma 3.3 and (4) is (3.5). Finally (5) comes from the identification of elements in $\left(\left(\mathscr{U}(\mathfrak{g}) /\left(\mathscr{U}(\mathfrak{g}) \cdot \mathfrak{g}^{\mu}\right)^{\otimes 2}\right)^{K^{\mu}}\right.$ with bidifferential operators.

Note that since both $F_{\hbar}(u, v)$ and $u *_{\hbar} v$ depend rationally on $\hbar$ (for fixed polynomials $u, v$ ) the proof implies in particular that these rational functions agree almost everywhere, hence must have the same poles.

\section{First convergence properties}

In the following we use the Karabegov description to see that any formal $K$-invariant star product $\star$ of Wick type with a nice Karabegov form is the Taylor expansion of a strict product on $\operatorname{Pol}\left(\mathcal{O}_{\mu}\right)$. We use the construction of Karabegov from Subsection 2.2 to obtain this strict product. To do this, we first need to prove a couple of technical lemmas.

For two roots $\alpha, \beta \in \Delta$ with $\alpha+\beta \in \Delta$ we define $N_{\alpha, \beta} \in \mathbb{C}$ by $\left[X_{\alpha}, X_{\beta}\right]=N_{\alpha, \beta} X_{\alpha+\beta}$. It is known that $N_{\alpha, \beta} \neq 0$, [25, Chapter VI.1, Theorem 6.6]. Recall that $v_{X}:=(-X)_{\mathcal{O}_{\mu}}:=\left.\frac{\mathrm{d}}{\mathrm{d} t}\right|_{t=0} \operatorname{Ad}_{\exp (-t X)} x$ for $x \in \mathcal{O}_{\mu}$ and $X \in \mathfrak{k}$.

Lemma 4.1 A K-invariant closed 2-form on $\mathcal{O}_{\mu}$ satisfies

$$
\omega\left(\left[X_{\alpha}, X_{\beta}\right], X_{\gamma}\right)+\omega\left(\left[X_{\beta}, X_{\gamma}\right], X_{\alpha}\right)+\omega\left(\left[X_{\gamma}, X_{\alpha}\right], X_{\beta}\right)=0 .
$$


Here we use the identifications from (2.6).

ProOF: For three fundamental vector fields $\left(X_{\alpha}\right)_{\mathcal{O}_{\mu}},\left(X_{\beta}\right)_{\mathcal{O}_{\mu}}$ and $\left(X_{\gamma}\right)_{\mathcal{O}_{\mu}}$ we calculate

$$
\begin{aligned}
0= & \mathrm{d} \omega\left(\left(X_{\alpha}\right)_{\mathcal{O}_{\mu}},\left(X_{\beta}\right)_{\mathcal{O}_{\mu}},\left(X_{\gamma}\right)_{\mathcal{O}_{\mu}}\right) \\
=\left(X_{\alpha}\right)_{\mathcal{O}_{\mu}} \omega\left(\left(X_{\beta}\right)_{\mathcal{O}_{\mu}},\left(X_{\gamma}\right)_{\mathcal{O}_{\mu}}\right)+\left(X_{\beta}\right)_{\mathcal{O}_{\mu}} \omega\left(\left(X_{\gamma}\right)_{\mathcal{O}_{\mu}},\left(X_{\alpha}\right)_{\mathcal{O}_{\mu}}\right) & \quad+\left(X_{\gamma}\right)_{\mathcal{O}_{\mu}} \omega\left(\left(X_{\alpha}\right)_{\mathcal{O}_{\mu}},\left(X_{\beta}\right)_{\mathcal{O}_{\mu}}\right)-\omega\left(\left[\left(X_{\alpha}\right)_{\mathcal{O}_{\mu}},\left(X_{\beta}\right)_{\mathcal{O}_{\mu}}\right],\left(X_{\gamma}\right)_{\mathcal{O}_{\mu}}\right) \\
& \quad-\omega\left(\left[\left(X_{\beta}\right)_{\mathcal{O}_{\mu}},\left(X_{\gamma}\right)_{\mathcal{O}_{\mu}}\right],\left(X_{\alpha}\right)_{\mathcal{O}_{\mu}}\right)-\omega\left(\left[\left(X_{\gamma}\right)_{\mathcal{O}_{\mu}},\left(X_{\alpha}\right)_{\mathcal{O}_{\mu}}\right],\left(X_{\beta}\right)_{\mathcal{O}_{\mu}}\right) \\
& \quad \omega\left(\left[X_{\alpha}, X_{\beta}\right]_{\mathcal{O}_{\mu}},\left(X_{\gamma}\right)_{\mathcal{O}_{\mu}}\right)+\omega\left(\left[X_{\beta}, X_{\gamma}\right]_{\mathcal{O}_{\mu}},\left(X_{\alpha}\right)_{\mathcal{O}_{\mu}}\right)+\omega\left(\left[X_{\gamma}, X_{\alpha}\right]_{\mathcal{O}_{\mu}},\left(X_{\beta}\right)_{\mathcal{O}_{\mu}}\right) .
\end{aligned}
$$

Here we used that since $\omega$ is $K$-invariant, its Lie derivative with respect to fundamental vector fields vanishes.

Lemma 4.2 Any K-invariant closed real $(1,1)$-form $\omega$ on a coadjoint orbit $\mathcal{O}_{\mu}$ can be written as $\omega=\omega_{\gamma}$ for some $K$-equivariant map $\gamma: \mathcal{O}_{\mu} \rightarrow \Omega \subseteq \mathfrak{k}^{*}$.

Proof: By $K$-equivariance it suffices to check the claim at $\mu$, by which we mean that there is a $K^{\mu}$-invariant element $\gamma(\mu) \in \mathfrak{k}^{*}$ such that $\left.\omega\right|_{\mu}=\left.\gamma^{*} \omega_{\Omega}\right|_{\mu}$, where $\gamma$ is the unique $K$-equivariant map evaluating to $\gamma(\mu)$ at $\mu$. Recall that at $\mu$ we have a basis $X_{\alpha}, \alpha \in \hat{\Delta}$ for the tangent space $T_{\mu}^{\mathbb{C}} \mathcal{O}_{\mu}$. Extend $\omega$ by zero on $\mathfrak{h}$ and the root spaces $\mathfrak{g}_{\alpha}$ with $\alpha \in \Delta^{\prime}$. Since we have

$$
\left.T_{x} \gamma v_{X}\right|_{x}=\left.T_{x} \gamma \frac{\mathrm{d}}{\mathrm{d} t}\right|_{t=0} \operatorname{Ad}_{\exp (-t X)} x=\left.\frac{\mathrm{d}}{\mathrm{d} t}\right|_{t=0} \operatorname{Ad}_{\exp (-t X)}^{*} \gamma(x)=\left.v_{X}^{\Omega}\right|_{\gamma(x)},
$$

we can immediately note that $\left.\gamma^{*} \omega_{\Omega}\right|_{\mu}\left(X_{\alpha}, X_{\beta}\right)=\left.\omega_{\Omega}\left(v_{X_{\alpha}}^{\Omega}, v_{X_{\beta}}^{\Omega}\right)\right|_{\gamma(\mu)}=\left\langle\gamma(\mu),\left[X_{\alpha}, X_{\beta}\right]\right\rangle$. Therefore there is an obvious candidate for $\gamma(\mu)$ : choose $\gamma(\mu) \in \mathfrak{h}^{*}$ (and extend it as zero on all root spaces) such that

$$
\left.\omega\right|_{\mu}\left(X_{\alpha}, X_{-\alpha}\right)=\left\langle\gamma(\mu),\left[X_{\alpha}, X_{-\alpha}\right]\right\rangle
$$

holds for all fundamental roots $\alpha$. For fundamental roots $\left[X_{\alpha}, X_{-\alpha}\right]$ is a basis of $\mathfrak{h}$, so this definition makes sense. Note also that $\omega$ is real, so $\gamma(\mu)$ really lies in $\mathfrak{k}^{*}$. We prove below that (4.3) already implies $\left.\omega\right|_{\mu}\left(X_{\alpha}, X_{\beta}\right)=\left\langle\gamma(\mu),\left[X_{\alpha}, X_{\beta}\right]\right\rangle=\left.\gamma^{*} \omega_{\Omega}\right|_{\mu}\left(X_{\alpha}, X_{\beta}\right)$ for all roots $\alpha, \beta$. Thereafter we show that $\gamma(\mu)$ is indeed $K^{\mu}$-invariant.

We prove $\left.\omega\right|_{\mu}\left(X_{\alpha}, X_{\beta}\right)=\left.\gamma^{*} \omega_{\Omega}\right|_{\mu}\left(X_{\alpha}, X_{\beta}\right)$ in increasing generality. First, note that by definition (4.3) it holds for a fundamental root $\alpha=-\beta$. A $K$-invariant form is also $\mathfrak{k}^{\mu}$-invariant at $\mu$, implying $\left.\omega\right|_{\mu}\left(\operatorname{ad}_{H} X_{\alpha}, X_{\beta}\right)+\left.\omega\right|_{\mu}\left(X_{\alpha}, \operatorname{ad}_{H} X_{\beta}\right)=0$ for all $H \in \mathfrak{h}$, so $\left.\omega\right|_{\mu}\left(X_{\alpha}, X_{\beta}\right)=0$ whenever $\alpha \neq-\beta$. Consequently $\left.\omega\right|_{\mu}\left(X_{\alpha}, X_{\beta}\right)$ and $\left.\gamma^{*} \omega_{\Omega}\right|_{\mu}\left(X_{\alpha}, X_{\beta}\right)$ also coincide whenever $\alpha \neq-\beta$. So the only remaining case is $\alpha=-\beta$ for non-fundamental roots. This follows from the following statement: if $\left.\omega\right|_{\mu}\left(X_{\alpha}, X_{-\alpha}\right)=\left.\gamma^{*} \omega_{\Omega}\right|_{\mu}\left(X_{\alpha}, X_{-\alpha}\right)$ holds for some roots $\alpha=\beta$ and $\alpha=\gamma$, then it also holds for $\beta+\gamma$ :

$$
\begin{aligned}
\left.\omega\right|_{\mu}\left(X_{\beta+\gamma}, X_{-\beta-\gamma}\right) & =\left.N_{\beta, \gamma}^{-1} \omega\right|_{\mu}\left(\left[X_{\beta}, X_{\gamma}\right], X_{-\beta-\gamma}\right) \\
& =-N_{\beta, \gamma}^{-1}\left(\left.\omega\right|_{\mu}\left(\left[X_{\gamma}, X_{-\beta-\gamma}\right], X_{\beta}\right)+\left.\omega\right|_{\mu}\left(\left[X_{-\beta-\gamma}, X_{\beta}\right], X_{\gamma}\right)\right) \\
& =-N_{\beta, \gamma}^{-1}\left(\left.N_{\gamma,-\beta-\gamma} \omega\right|_{\mu}\left(X_{-\beta}, X_{\beta}\right)+\left.N_{-\beta-\gamma, \beta} \omega\right|_{\mu}\left(X_{-\gamma}, X_{\gamma}\right)\right. \\
& =N_{\beta, \gamma}^{-1}\left(N_{\gamma,-\beta-\gamma}\left\langle\gamma(\mu), H_{\beta}\right\rangle+N_{-\beta-\gamma, \beta}\left\langle\gamma(\mu), H_{\gamma}\right\rangle\right. \\
& =\left\langle\gamma(\mu), N_{\beta, \gamma}^{-1}\left(N_{\gamma,-\beta-\gamma} H_{\beta}+N_{-\beta-\gamma, \beta} H_{\gamma}\right)\right\rangle \\
& =\left\langle\gamma(\mu),-H_{-\beta-\gamma}\right\rangle \\
& =\left.\gamma^{*} \omega_{\Omega}\right|_{\mu}\left(X_{\beta+\gamma}, X_{-\beta-\gamma}\right) .
\end{aligned}
$$


We used Lemma 4.1 in the second step and that the Jacobi identity implies $N_{\alpha, \beta} H_{\gamma}+N_{\beta, \gamma} H_{\alpha}+$ $N_{\gamma, \alpha} H_{\beta}=0$ in the second to last step.

Now let us prove $K^{\mu}$-invariance. Since $K^{\mu}$ is the centralizer of a torus and thus connected, it suffices to check $\mathfrak{k}^{\mu}$-invariance of $\gamma(\mu)$. Firstly, $\mathfrak{h}$-invariance is clear since $\gamma(\mu) \in \mathfrak{h}^{*}$. Secondly, if $\alpha \in \Delta^{\prime}$ then $\left\langle\operatorname{ad}_{X_{\alpha}}^{*} \gamma(\mu), Z\right\rangle=\left\langle\gamma(\mu),\left[-X_{\alpha}, Z\right]\right\rangle$, which vanishes whenever $Z \in \mathfrak{h}$ or any of the root spaces $\mathfrak{g}_{\beta}$ with $\beta \neq-\alpha$. Also $\left\langle\gamma(\mu),\left[X_{\alpha}, X_{-\alpha}\right]\right\rangle=\left.\gamma^{*} \omega_{\Omega}\right|_{\mu}\left(X_{\alpha}, X_{-\alpha}\right)=\left.\omega\right|_{\mu}\left(X_{\alpha}, X_{-\alpha}\right)=0$.

Theorem 4.3 Let $\mathcal{O}_{\mu}$ be an adjoint orbit with a formal differential $K$-invariant star product $\star$ of Wick type. Assume that its Karabegov form $\omega=\sum_{r \in \mathbb{N}_{0}} z^{r} \omega_{r}$ is the Taylor series around zero of some rational real form $\omega(\hbar)$, regular at zero. Then for any $p, q \in \operatorname{Pol}\left(\mathcal{O}_{\mu}\right)$ and $x \in \mathcal{O}_{\mu},(p \star q)(x)$ is the Taylor series of a unique rational function $\left(p *_{\hbar} q\right)(x)$ in $\hbar$ having only finitely many poles that might depend on $p$ and $q$ but not on $x$. For all $p, q \in \operatorname{Pol}\left(\mathcal{O}_{\mu}\right)$ and $\hbar$ not lying in this set of poles $p *_{\hbar} q$ is again a polynomial.

Proof: It is known (see e.g. [30]) that the Karabegov form of a $K$-invariant star product is $K$ invariant, i.e. all $\omega_{r}$ are $K$-invariant. Thus $\omega(\hbar)=\sum_{j} b_{j}(\hbar) \omega^{j}$ is also $K$-invariant, so we can assume that the $\omega^{j}$ are $K$-invariant. Here the $b_{j}$ are rational functions of $\hbar$, regular at 0 . Since the star product is also of Wick type, we get from Lemma 4.2 that all $\omega^{j}$ are of the form $\omega_{\gamma^{j}}$ for some maps $\gamma^{j}: \mathcal{O}_{\mu} \rightarrow \mathfrak{k}^{*}$. Thus the corresponding $K$-equivariant family $f_{\bullet}^{(\hbar)}=\sum_{j} b_{j}(\hbar) f_{\bullet}^{j}$ is rational and regular at 0 , so can be used to construct Karabegov's star product $*_{\hbar}$. Since for polynomials their product $\left(f *_{\hbar} g\right)(x)$ is a rational function without pole at $\hbar=0$ by Theorem 2.9, , ), its Taylor series coincides with $\left(f \star^{\prime} g\right)(x)$, where $\star^{\prime}$ is the formal star product associated to $*_{\hbar}$. By construction its Karabegov form is the Taylor series expansion of $\omega(\hbar)$, thus coincides with the Karabegov form of $\star$. Consequently $\star=\star^{\prime}$. All claims now follow easily from Karabegov's construction.

This result establishes the existence of a subalgebra, namely the polynomials, on which we obtain associative products for all complex numbers $\hbar$ (that are different from the poles), thereby passing from the formal to the strict setting. In the next subsection, we show how this subalgebra can be enlarged in the case of the 2-sphere.

\section{Continuity of the star product on $\mathbb{\$}^{2}$}

In this section we focus on the particular case of the two dimensional sphere $\mathbb{S}^{2}$ interpreted as a coadjoint orbit of $\mathrm{SU}(2)$. Let $\mathfrak{s l}_{2}(\mathbb{C})$ be the Lie algebra of trace-free complex $2 \times 2$ matrices with standard basis denoted by $(H, X, Y)$. Consider $\mathfrak{h}=\langle H\rangle$. The compact real form $\mathfrak{k}$ of $\mathfrak{s l}_{2}(\mathbb{C})$ is $\mathfrak{s u}_{2}$ and the Killing form is given by

$$
B(H, H)=8, \quad B(X, Y)=B(Y, X)=4 \quad \text { and } \quad B(H, X)=B(H, Y)=B(X, X)=B(Y, Y)=0 .
$$

Note that on the dual of $\mathfrak{s l}_{2}(\mathbb{C})$ we have the dual basis to $(H, X, Y)$ denoted by $\left(H^{*}, X^{*}, Y^{*}\right)$ and another basis obtained by using the isomorphism of $\mathfrak{s l}_{2}(\mathbb{C})$ and $\mathfrak{s l}_{2}(\mathbb{C})^{*}$ induced by the Killing form,

$$
\left(H^{b}, X^{b}, Y^{b}\right)=\left(8 H^{*}, 4 Y^{*}, 4 X^{*}\right) .
$$

Here and in the following $Z^{\mathrm{b}}=B(Z, \cdot) \in \mathfrak{s l}_{2}(\mathbb{C})^{*}$ for $Z \in \mathfrak{s l}_{2}(\mathbb{C})$.

The complex connected simply-connected Lie group corresponding to $\mathfrak{s l}_{2}(\mathbb{C})$ is the special linear group $\mathrm{SL}(2, \mathbb{C})$ and the closed connected subgroup with Lie algebra $\mathfrak{s u}_{2}$ is the special unitary group $\mathrm{SU}(2)$. Elements $k \in \mathrm{SU}(2)$ can be explicitly parametrized by

$$
k=\left(\begin{array}{cc}
u & -\bar{v} \\
v & \bar{u}
\end{array}\right) \quad \text { with } \quad u, v \in \mathbb{C} \quad \text { satisfying } \quad|u|^{2}+|v|^{2}=1 .
$$


Thus, it is not hard to show that adjoint orbits of $\mathrm{SU}(2)$ coincide with spheres (with respect to the inner product given by the Killing form).

We identify linear functionals $\alpha \in \mathfrak{h}^{*}$ which are purely imaginary on $\mathfrak{h} \cap \mathfrak{s u}_{2}$ with real numbers $\lambda_{\alpha}:=\alpha(H) \in \mathbb{R}$ by evaluating them at $H$. Weights correspond to integers under this identification. Excluding the trivial adjoint orbit $\{0\}$, all other orbits are spheres with non-zero radius and intersect $\mathfrak{h}$ in exactly two points $\mu$ and $-\mu$. We choose $\mu$ such that $\lambda:=\lambda_{B(-\mathrm{i} \mu, \cdot)}=B(-\mathrm{i} \mu, H)$ is positive. If $\mu=\mathrm{i} H$ then $B(-\mathrm{i} \mu, \cdot)=H^{b}=8 H^{*}$ and $\lambda=8$. Denote such an orbit by $\mathbb{S}_{\lambda}^{2}$. Using this convention the map $\psi_{\mu}$ from Section 3 is given by

$$
\psi_{\mu}(k)=\operatorname{Ad}_{k} \mu=\frac{\mathrm{i} \lambda}{8}((u \bar{u}-v \bar{v}) H+2 u \bar{v} X+2 \bar{u} v Y) .
$$

The roots are given by $\alpha_{+}, \alpha_{-}$with $\lambda_{\alpha_{+}}=2$ and $\lambda_{\alpha_{-}}=-2$. There are exactly two SU(2)-invariant complex structures on the sphere, depending on which of these roots we define positive. The following lemma is a consequence of Proposition 2.3. Note that $H_{\alpha_{+}}=H$ and so $\lambda\left(H_{\alpha_{+}}\right)>0$.

Lemma 5.1 The Kähler complex structure $J_{\text {Kähler }}$ on the sphere corresponds to an ordering for which $\alpha_{-}$is positive.

Remember that to obtain a star product of Wick type, we need to choose $J$ opposite to $J_{\text {Kähler }}$, so in the corresponding ordering $\alpha_{+}$is positive.

We observe that any $K$-invariant 2 -form on the sphere is a scalar multiple of the KKS form $\omega_{\mathbb{S}^{2}}$ : by $K$-invariance it is determined by its value $\left.\alpha\right|_{\mu}$ and the space of 2 -forms on a two dimensional vector space is one dimensional. In particular, any rational $K$-invariant 2 -form which is regular at zero is given by $\omega(\hbar)=\zeta(\hbar) \omega_{\mathbb{S}^{2}}$ with a rational function $\zeta$ having no pole at zero. Without loss of generality we only consider the formal star product $\star$ with $\omega=\omega_{\mathbb{S}^{2}}$ and the associated product $*_{\hbar}$. One checks easily that the associated $K$-equivariant family is $f_{Z}:=Z^{b}: \mathbb{S}_{\lambda}^{2} \rightarrow \mathbb{C}$. (For better readability we leave out the restriction of $Z^{b}$ to $\mathbb{S}_{\lambda}^{2}$.)

We would like to construct a topology on the polynomials such that the product $*_{\hbar}$ becomes continuous. For this, we need some explicit estimates of the coefficients of the star product. Since we have already seen that the constructions of Karabegov and Alekseev-Lachowska agree we can work with either of them. We will mostly use the construction of Alekseev-Lachowska since it produces easier formulas, but in the last part of this section we describe how to obtain the same results in the Karabegov approach.

\subsection{Formulas for $\mathrm{SU}(2)$ and related properties}

In this section we introduce notation and a locally convex topology, called the $T_{R}$-topology, on the polynomials on the sphere. Both are needed in the next section to prove the continuity of $*_{\hbar}$.

As a first step we recall a formula, obtained by Alekseev and Lachowska in [1, for the star product on the two sphere. With our conventions $\mathfrak{n}^{+}=\langle X\rangle$ and $\mathfrak{n}^{-}=\langle Y\rangle$ with $X$ of degree 1 and $Y$ of degree -1 . The pairing is diagonal with respect to the bases $\left\{1, X, X^{2}, \ldots\right\}$ of $\mathscr{U}\left(\mathfrak{n}^{+}\right)$and $\left\{1, Y, Y^{2}, \ldots\right\}$ of $\mathscr{U}\left(\mathfrak{n}^{-}\right)$due to degree reasons, so we just have to calculate the normalization.

Lemma 5.2 We have $\left(X^{n} Y^{n}\right)_{0}=n ! H(H-1) \ldots(H-n+1)$.

ProOF: We prove this by induction and a simple calculation:

$$
\left(X^{n} Y^{n}\right)_{0}=(\underbrace{Y X^{n} Y^{n-1}}_{(\ldots)_{0}=0}+\sum_{i=0}^{n-1} X^{i} H X^{n-1-i} Y^{n-1})_{0}
$$




$$
\begin{aligned}
& =\left(\sum_{i=0}^{n-1}\left(H X^{i}+\sum_{j=0}^{i-1} X^{j}(-2 X) X^{i-j-1}\right) X^{n-1-i} Y^{n-1}\right)_{0} \\
& =\left(\sum_{i=0}^{n-1} H X^{n-1} Y^{n-1}-2 \sum_{i=0}^{n-1} \sum_{j=0}^{i-1} X^{n-1} Y^{n-1}\right)_{0} \\
& =\left(n H X^{n-1} Y^{n-1}-n(n-1) X^{n-1} Y^{n-1}\right)_{0} \\
& =n(H-n+1)\left(X^{n-1} Y^{n-1}\right)_{0} .
\end{aligned}
$$

The previous lemma implies immediately that

$$
\left(Y^{n}, X^{n}\right)_{\hbar}=\left\langle\frac{B(-\mathrm{i} \mu, \cdot)}{\hbar},(-1)^{n}\left(X^{n} Y^{n}\right)_{0}\right\rangle=(-1)^{n} n ! \frac{\lambda}{\hbar}\left(\frac{\lambda}{\hbar}-1\right) \cdots\left(\frac{\lambda}{\hbar}-(n-1)\right)
$$

and therefore the pairing $(\cdot, \cdot)_{\hbar}$ is non-degenerate if $\hbar \notin \Omega:=\left\{0, \lambda, \frac{\lambda}{2}, \frac{\lambda}{3}, \ldots\right\}$. Consequently, for $\hbar \notin \Omega$ the product $p *_{\hbar} q=F_{\hbar}(p, q)$ is well defined for all $p, q \in \operatorname{Pol}\left(\mathbb{S}_{\lambda}^{2}\right)$, where

$$
F_{\hbar}=\sum_{n=0}^{\infty} \frac{(-1)^{n} \hbar^{n}}{n ! \lambda(\lambda-\hbar) \cdots(\lambda-(n-1) \hbar)} Y^{n} \otimes X^{n} .
$$

Note that the stabilizer Lie group of $\mu$ is $\mathrm{SU}(2)^{\mu}=\left\{\operatorname{diag}\left(\mathrm{e}^{\mathrm{i} t}, \mathrm{e}^{-\mathrm{i} t}\right) \mid t \in \mathbb{R}\right\} \cong \mathrm{U}(1)$. Therefore $\mathbb{S}_{\lambda}^{2} \cong \mathrm{SU}(2) / \mathrm{U}(1)$ and we can identify smooth functions on $\mathbb{S}_{\lambda}^{2}$ with smooth functions on $\mathrm{SU}(2)$, which are invariant under the $\mathrm{U}(1)$-action on $\mathrm{SU}(2)$ by right multiplication, i.e. $f(x)=f(x y)$ for $x \in \mathrm{SU}(2)$ and $y \in \mathrm{U}(1)$. Here elements of $\mathrm{U}(1)$ are realized as the $2 \times 2$-matrices from above. We will refer to the invariance property simply by right-invariance in the following and write $\mathscr{C}_{\mathbb{C}}^{\infty}(\mathrm{SU}(2))^{\mathrm{U}(1)}$ for the space of such functions.

Note that $Y^{n} \otimes X^{n}$ in (5.5) acts on the extensions $\hat{p}, \hat{q}$ of two polynomials $p, q$ on the adjoint orbit to right invariant functions on $\mathrm{SU}(2)$ by the left invariant bidifferential operators corresponding to $Y^{n} \otimes X^{n}$, giving another right invariant function $\left(Y^{\text {left }}\right)^{n} \hat{p} \cdot\left(X^{\text {left }}\right)^{n} \hat{q}$ and thus also a polynomial on the orbit. However, neither $\left(Y^{\text {left }}\right)^{n} \hat{p}$ nor $\left(X^{\text {left }}\right)^{n} \hat{q}$ is right invariant in general, so that these functions do not necessarily descend to well-defined functions on the adjoint orbit. It is just their product $\left(Y^{\text {left }}\right)^{n} \hat{p} \cdot\left(X^{\text {left }}\right)^{n} \hat{q}$ that does.

To obtain continuity estimates it is very convenient to cure this pathology by introducing a bigger class of functions than right invariant extensions of polynomials. In this class $\left(Y^{\text {left }}\right)^{n}$ should be well-defined and the $n$-fold composition of $Y^{\text {left }}$. Indeed, such a class of functions exists and is given by $K$-finite functions. Remember that a function $f$ on a manifold $M$ with an action of $K$ is $K$-finite, if the span of $k \triangleright f$ is finite dimensional. (Here $k \triangleright f(m)=f\left(k^{-1} \triangleright m\right.$ ) for $k \in K$ and $m \in M$.) We use the following standard facts on $K$-finite functions.

Proposition 5.3 Let $K$ be a compact connected semisimple Lie group.

i.) The $K$-finite functions on an adjoint orbit $K / K^{\mu}$ coincide with polynomials on the adjoint orbit.

ii.) If $K$ is realized as a matrix Lie group, then the $K$-finite functions on $K$ coincide with polynomials of the matrix entries and their complex conjugates.

PROOF: It is a standard argument, see e.g. [22, Lemma 15].

Definition 5.4 (The algebras $\mathscr{P}_{\mathrm{SU}(2)}$ and $\mathscr{R}_{\mathrm{SU}(2)}$ ) The algebra $\mathscr{P}_{\mathrm{SU}(2)}$ is defined to be the algebra generated by the functions $U, \bar{U}, V, \bar{V}: \mathrm{SU}(2) \rightarrow \mathbb{C}$, given for $k \in \mathrm{SU}(2)$ by

$$
U: k \mapsto u, \quad \bar{U}: k \mapsto \bar{u}, \quad V: k \mapsto v, \quad \bar{V}: k \mapsto \bar{v} .
$$


The algebra $\mathscr{R}_{\mathrm{SU}(2)}$ is defined to be the algebra generated by the functions $A, B, C: \mathrm{SU}(2) \rightarrow \mathbb{C}$, given for $k \in \mathrm{SU}(2)$ by

$$
A=U \bar{U}-V \bar{V}: k \mapsto|u|^{2}-|v|^{2}, \quad B=\bar{U} V: k \mapsto \bar{u} v \quad \text { and } \quad C=U \bar{V}: k \mapsto u \bar{v} .
$$

From Proposition 5.3 it is clear that $\mathscr{P}_{\mathrm{SU}(2)}$ are just the $\mathrm{SU}(2)$-finite functions on $\mathrm{SU}(2)$. We will see below that $\mathscr{R}_{\mathrm{SU}(2)}$ are the right invariant elements of $\mathscr{P}_{\mathrm{SU}(2)}$.

Note that $U \bar{U}+V \bar{V}=1$. We would also like to consider a "free" commutative algebra without this relation. To this end define $\hat{U}, \hat{\bar{U}}, \hat{V}, \hat{\bar{V}}, \hat{A}, \hat{B}, \hat{C}, \hat{D}: \mathbb{C}^{2} \rightarrow \mathbb{C}$ by

$$
\hat{U}:\left(z_{1}, z_{2}\right) \mapsto z_{1}, \quad \hat{\bar{U}}:\left(z_{1}, z_{2}\right) \mapsto \bar{z}_{1}, \quad \hat{V}:\left(z_{1}, z_{2}\right) \mapsto z_{2} \quad \text { and } \quad \hat{\bar{V}}:\left(z_{1}, z_{2}\right) \mapsto \bar{z}_{2} .
$$

$\hat{A}, \hat{B}$ and $\hat{C}$ are the same products as before, just decorating (5.7) with hats and $\hat{D}=\hat{U} \hat{\bar{U}}+\hat{V} \hat{\bar{V}}$. Note that the polynomial algebra $\mathscr{P}_{\mathbb{C}^{2}}$ on $\mathbb{C}^{2}$ is generated by $\hat{U}, \hat{\bar{U}}, \hat{V}$ and $\hat{\bar{V}}$ and denote the algebra generated by $\hat{A}, \hat{B}, \hat{C}$ and $\hat{D}$ by $\mathscr{R}_{\mathbb{C}^{2}}$.

Recall that $\mathrm{SU}(2)^{\mu}=\left\{\operatorname{diag}\left(\mathrm{e}^{\mathrm{i} t}, \mathrm{e}^{-\mathrm{i} t}\right) \mid t \in \mathbb{R}\right\}$. For a monomial $U^{\alpha} \bar{U}^{\beta} V^{\gamma} \bar{V}^{\delta}$ we introduce the numbers $n=\alpha+\gamma$ and $\bar{n}=\beta+\delta$. Then a monomial in $U, \bar{U}, V$ and $\bar{V}$ is right invariant if and only if $n=\bar{n}$. Consider the action of $\mathrm{U}(1)$ on $\mathbb{C}^{2}$ by componentwise multiplication and the induced action on $\mathscr{P}_{\mathbb{C}^{2}}$. Defining $\hat{n}$ and $\hat{n}$ in the same way as $n$ and $\bar{n}$, the right invariant monomials are exactly the ones with $\hat{n}=\hat{\bar{n}}$. So this says that the right invariant elements of $\mathscr{P}_{\mathbb{C}^{2}}$ are exactly $\mathscr{R}_{\mathbb{C}^{2}}$ and the ones of $\mathscr{P}_{\mathrm{SU}(2)}$ are $\mathscr{R}_{\mathrm{SU}(2)}$.

Proposition 5.5 $\mathscr{R}_{\mathrm{SU}(2)}$ is isomorphic to $\operatorname{Pol}\left(\mathbb{S}_{\lambda}^{2}\right)$.

ProOF: We only need to see that the restriction and extension maps between right invariant functions on $\mathrm{SU}(2)$, i.e. $\mathscr{C}_{\mathbb{C}}^{\infty}(\mathrm{SU}(2))^{\mathrm{U}(1)}$, and functions on the orbit, i.e. $\mathscr{C}_{\mathbb{C}}^{\infty}(\mathrm{SU}(2) / \mathrm{U}(1))$, map $\mathscr{R}_{\mathrm{SU}(2)}$ to $\operatorname{Pol}\left(\mathbb{S}_{\lambda}^{2}\right)$ and vice versa. However, this is immediately clear from Proposition 5.3 and since by $\mathrm{SU}(2)$-equivariance of the map $\mathrm{SU}(2) \rightarrow \mathrm{SU}(2) / \mathrm{U}(1)$ they map $\mathrm{SU}(2)$-finite functions to $\mathrm{SU}(2)$-finite functions.

We can determine the isomorphism explicitly: from (5.4) it follows that

$$
H^{\mathrm{b}} \circ \psi_{\lambda}=\mathrm{i} \lambda A, \quad X^{\mathrm{b}} \circ \psi_{\lambda}=\mathrm{i} \lambda B \quad \text { and } \quad Y^{\mathrm{b}} \circ \psi_{\lambda}=\mathrm{i} \lambda C .
$$

It is well-known that any polynomial vanishing on the 3 -sphere viewed as a submanifold of $\mathbb{R}^{4}$ is a multiple of $x_{1}^{2}+x_{2}^{2}+x_{3}^{2}+x_{4}^{2}-1$, where $\left(x_{1}, x_{2}, x_{3}, x_{4}\right)$ are standard coordinates on $\mathbb{R}^{4}$. This implies that any element of $\mathscr{P}_{\mathbb{C}^{2}}$ vanishing on $\mathrm{SU}(2) \subseteq \mathbb{C}^{2 \times 2}$ (from $p \in \mathscr{P}_{\mathbb{C}^{2}}$ we obtain a function on $\mathrm{SU}(2)$ by composing with the map projecting a $2 \times 2$ matrix to its first column) if and only if it is a multiple of $\hat{U} \hat{\bar{U}}+\hat{V} \hat{\bar{V}}-1$. Similarly an element of $\mathbb{C}[\hat{A}, \hat{B}, \hat{C}]$, the algebra generated by $\hat{A}, \hat{B}$ and $\hat{C}$, vanishes on $\mathrm{SU}(2)$ if and only if it is a multiple of $\hat{A}^{2}+4 \hat{B} \hat{C}-1$. Consequently, we can prove the following claim.

Proposition 5.6 We have

$$
\mathscr{P}_{\mathrm{SU}(2)} \cong \mathscr{P}_{\mathbb{C}^{2}} /\langle\hat{U} \hat{\bar{U}}+\hat{V} \hat{\bar{V}}-1\rangle \quad \text { and } \quad \mathscr{R}_{\mathrm{SU}(2)} \cong \mathscr{R}_{\mathbb{C}^{2}} /\langle\hat{U} \hat{\bar{U}}+\hat{V} \hat{\bar{V}}-1\rangle \cong \mathbb{C}[\hat{A}, \hat{B}, \hat{C}] /\left\langle\hat{A}^{2}+4 \hat{B} \hat{C}-1\right\rangle
$$

Now since $X^{\text {left }}$ and $Y^{\text {left }}$ are left invariant vector fields, it is clear that by applying them to a $\mathrm{SU}(2)$-finite function, we get a $\mathrm{SU}(2)$-finite function again.

Lemma 5.7 We have:

$$
\begin{array}{llll}
X^{\text {left }} U=0, & X^{\text {left }} V=0, & X^{\text {left }} \bar{U}=V, & X^{\text {left }} \bar{V}=-U, \\
Y^{\text {left }} U=-\bar{V}, & Y^{\text {left }} V=\bar{U}, & Y^{\text {left }} \bar{U}=0, & Y^{\text {left }} \bar{V}=0 .
\end{array}
$$


ProOF: Let us compute:

$$
\begin{aligned}
X^{\mathrm{left}} \bar{V}(k) & =\left.\frac{\mathrm{d}}{\mathrm{d} t}\right|_{t=0} \exp \left(t\left(\begin{array}{ll}
0 & 1 \\
0 & 0
\end{array}\right)\right) \triangleright \bar{V}(k) \\
& =\left.\frac{\mathrm{d}}{\mathrm{d} t}\right|_{t=0} \bar{V}\left(\left(\begin{array}{cc}
u & v \\
-\bar{v} & \bar{u}
\end{array}\right)\left(\begin{array}{ll}
1 & t \\
0 & 1
\end{array}\right)\right) \\
& =\left.\frac{\mathrm{d}}{\mathrm{d} t}\right|_{t=0} \bar{V}\left(\begin{array}{cc}
u & u t+v \\
-\bar{v} & -t \bar{v}+\bar{u}
\end{array}\right) \\
& =-\left.\frac{\mathrm{d}}{\mathrm{d} t}\right|_{t=0}(u t+v) \\
& =-u \\
& =-U(k) .
\end{aligned}
$$

The other equalities are obtained similarly.

It will be convenient in the following to not just have a product on $\mathscr{R}_{\mathrm{SU}(2)}$, but also on $\mathscr{P}_{\mathbb{C}^{2}}$ and $\mathscr{R}_{\mathbb{C}^{2}}$. Thus define $*_{\hbar}^{\mathscr{P}}: \mathscr{P}_{\mathbb{C}^{2}} \times \mathscr{P}_{\mathbb{C}^{2}} \rightarrow \mathscr{P}_{\mathbb{C}^{2}}$ by applying the element $F_{\hbar}$ from (5.5), with the derivatives defined by decorating (5.11a) and (5.11b) with hats. It is clear from the construction that $*_{\hbar}^{\mathscr{P}}$ restricts to a product $*_{\hbar}^{\mathscr{R}}$ on $\mathscr{R}_{\mathbb{C}^{2}}$ and descends to $\mathscr{R}_{\mathrm{SU}(2)}$, where it coincides with $*_{\hbar}$. Keep in mind that $*_{\hbar}^{\mathscr{P}}$ is not associative any more, but that the associativity only holds on the quotient.

Lemma 5.8 Let $p=p_{1} \cdots p_{d}$ be a monomial with $p_{i} \in\{A, B, C\}$. Let $Z$ be any of $U, \bar{U}, V$ or $\bar{V}$. Then

$$
\begin{aligned}
& \text { i.) }\left(X^{\text {left }}\right)^{2} Z=\left(Y^{\text {left }}\right)^{2} Z=0 . \\
& \text { ii.) }\left(X^{\text {left }}\right)^{n} p=\left(Y^{\text {left }}\right)^{n} p=0 \text { if } n>d .
\end{aligned}
$$

Proof: Part i .) follows immediately from (5.11a ) and (5.11b). Then using the product rule it follows that $\left(X^{\text {left }}\right)^{2} p_{i}=0$ since at least one of $X^{\text {left }} Z$ or $X^{\text {left }} \bar{Z}$ vanishes and similarly for $Y$. This implies part [ii.) by using the product rule again.

Let $V$ be a finite dimensional locally convex vector space and $R \in \mathbb{R}$. For any continuous seminorm $p$ define a seminorm on the tensor algebra $T^{\bullet}(V)$ by

$$
p_{R}=\sum_{n=0}^{\infty}(n !)^{R} p^{n}
$$

where $p^{n}=p \otimes \ldots \otimes p$. We write $T_{R}^{\bullet}(V)$ for the locally convex space that arises if $T^{\bullet}(V)$ is endowed with the seminorms $p_{R}$ for all continuous seminorms $p$. We get an induced topology on the subspace $\mathrm{S}^{\bullet}(V)$ that is closed if $R \geq 0$. In this case the evaluation functional $\delta_{\alpha}: \mathrm{S}_{R}^{\bullet}(V) \rightarrow \mathbb{C}$ at $\alpha \in V^{*}$ is continuous.

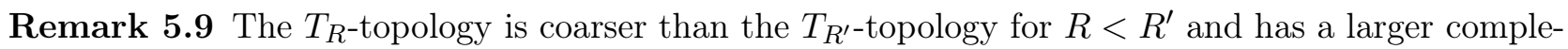
tion, see e.g. [39, Lemma 3.6]. Thus we are mainly interested in the case $R=0$ below, even though we formulate our results for all $R \geq 0$. The statements above remain true in infinite dimensions if one uses the projective tensor product.

For a finite dimensional vector space all norms are equivalent and thus we can fix any norm $\|\cdot\|$ and it suffices to consider the seminorms $(C\|\cdot\|)_{R}$ for all $C \geq 1$. In the following we will have to estimate these seminorms. The tensor product of 1 -norms has a particularly easy form. 
Lemma 5.10 Let $V$ be a finite dimensional vector space with basis $e_{1}, \ldots, e_{n}$. Let $\|\cdot\|_{1}$ denote the usual 1-norm with respect to this basis, i.e. if $v \in V, v=\sum_{i} v_{i} e_{i}$ then $\|v\|_{1}=\sum_{i}\left|v_{i}\right|$. Then on the tensor product $V^{\otimes k}$ we have

$$
\|\cdot\|_{1} \otimes \ldots \otimes\|\cdot\|_{1}(t)=\sum_{i_{1}, \ldots, i_{k}=1}^{n}\left|t_{i_{1} \cdots i_{k}}\right| \quad \text { if } \quad t=\sum_{i_{1}, \ldots, i_{k}=1}^{n} t_{i_{1} \ldots i_{k}} e_{i_{1}} \otimes \cdots \otimes e_{i_{k}} .
$$

We write the symmetric tensor product as a simple product and use the convention that $x_{1} \cdots x_{n}=$ $\frac{1}{n !} \sum_{\sigma \in S_{n}} x_{\sigma(1)} \otimes \ldots \otimes x_{\sigma(n)}$, so we have

$$
\left(C\|\cdot\|_{1}\right)_{R}\left(\sum_{I \in \mathbb{N}_{0}^{n}} a_{I} e_{1}^{I_{1}} \ldots e_{n}^{I_{n}}\right)=\sum_{I \in \mathbb{N}_{0}^{n}}(|I| !)^{R} C^{|I|}\left|a_{I}\right| .
$$

Here we use multiindices $I=\left(I_{1}, \ldots, I_{n}\right) \in \mathbb{N}_{0}^{n}$ and $|I|=I_{1}+\cdots+I_{n}$.

Corollary 5.11 Let $V$ be a finite dimensional vector space with basis $e_{1}, \ldots, e_{n}$ and $\|\cdot\|_{1}$ the 1-norm with respect to this basis. Then to prove continuity of a linear map $f: S_{R}^{\bullet}(V) \rightarrow W$ where $W$ is some locally convex vector space, it suffices to find for each continuous seminorm $p$ on $W$ a constant $C_{p}>0$ such that

$$
p\left(f\left(e_{1}^{I_{1}} \ldots e_{n}^{I_{n}}\right)\right) \leq\left(C_{p}\|\cdot\|_{1}\right)_{R}\left(e_{1}^{I_{1}} \ldots e_{n}^{I_{n}}\right)
$$

holds for any multiindex $I=\left(I_{1}, \ldots, I_{n}\right) \in \mathbb{N}_{0}^{n}$.

Proof: It follows from (5.15) and Lemma 5.10 that

$$
\begin{aligned}
p\left(f\left(\sum_{I \in \mathbb{N}_{0}^{n}} a_{I} e_{1}^{I_{1}} \ldots e_{n}^{I_{n}}\right)\right) & \leq \sum_{I \in \mathbb{N}_{0}^{n}}\left|a_{I}\right| p\left(f\left(e_{1}^{I_{1}} \ldots e_{n}^{I_{n}}\right)\right) \\
& \leq \sum_{I \in \mathbb{N}_{0}^{n}}\left|a_{I}\right|\left(C_{p}\|\cdot\|_{1}\right)_{R}\left(e_{1}^{I_{1}} \ldots e_{n}^{I_{n}}\right) \\
& =\left(C_{p}\|\cdot\|_{1}\right)_{R}\left(\sum_{I \in \mathbb{N}_{0}^{n}} a_{I} e_{1}^{I_{1}} \ldots e_{n}^{I_{n}}\right),
\end{aligned}
$$

where we assume that only finitely many coefficients $a_{I}$ are non-zero, so that all sums are in fact finite.

We need the following well-known estimate.

Lemma 5.12 For a fixed compact disc $D_{r}\left(z_{0}\right)=\left\{z \in \mathbb{C}|| z-z_{0} \mid \leq r\right\}$ with $D_{r}\left(z_{0}\right) \cap \mathbb{N}_{0}=\emptyset$ there are constants $0<C_{-} \leq 1 \leq C_{+}$depending on $r$ and $z_{0}$ such that for all $\delta \in \mathbb{N}$ and $z \in D_{r}\left(z_{0}\right)$ we have

$$
\delta ! C_{-}^{\delta} \leq|z(z-1) \cdots(z-\delta+1)| \leq \delta ! C_{+}^{\delta} .
$$

There are several ways to obtain continuity estimates for $*_{\hbar}$ and it is not quite clear which one is best suited for generalization. Therefore we define two topologies below, that turn out to be equivalent but can both be used to obtain continuity estimates.

Definition 5.13 (Reduction and quotient- $\boldsymbol{T}_{\boldsymbol{R}}$-topologies) $\quad$ i.) Let $\mathfrak{p}$ be the 1-norm with respect to the basis $\{\hat{U}, \hat{V}, \hat{\bar{U}}, \hat{\bar{V}}\}$ of $\left(\mathbb{C}^{2}\right)^{*}$ (thinking of $\mathbb{C}^{2}$ as a real vector space). The corresponding $T_{R}$-topology on $\mathrm{S}^{\bullet}\left(\left(\mathbb{C}^{2}\right)^{*}\right) \cong \mathscr{P}_{\mathbb{C}^{2}}$ induces a subspace topology on $\mathscr{R}_{\mathbb{C}^{2}}$ and thus a quotient topology on $\mathscr{R}_{\mathrm{SU}(2)}$, called the reduction- $T_{R}$-topology. 
ii.) Similarly, the 1-norm $\|\cdot\|_{1}$ with respect to the basis $\left\{H^{b}, X^{b}, Y^{b}\right\}$ on $\mathfrak{s l}_{2}(\mathbb{C})^{*}$ induces a $T_{R}$-topology on $\mathrm{S}^{\bullet}\left(\mathfrak{s l}_{2}(\mathbb{C})^{*}\right) \cong \operatorname{Pol}\left(\mathfrak{s l}_{2}(\mathbb{C})\right)$ and we call its quotient topology on $\operatorname{Pol}\left(\mathfrak{s l}_{2}(\mathbb{C})\right) / \mathscr{I}_{\text {van }}$ the quotient$T_{R}$-topology.

Here $\mathscr{I}_{\text {van }}$ is the ideal of polynomials vanishing on the orbit, which is generated by $\left(H^{b}\right)^{2}+X^{b} Y^{b}+\lambda^{2}$.

Proposition 5.14 The reduction- $T_{R}$-topology and quotient- $T_{2 R}$-topology agree.

Proof: Recall from Proposition 5.5 that the space of polynomials on the sphere is isomorphic to $\mathscr{R}_{\mathrm{SU}(2)}$ with the isomorphism given as in (5.9). Let $W$ be the three dimensional vector space spanned by $\hat{A}, \hat{B}$ and $\hat{C}$, endowed with the 1-norm $\|\cdot\|_{1}^{\prime}$ with respect to this basis. Consider the tensor algebra $S^{\bullet}(W)$ with the induced $T_{R}$-topology, then it is clear from Proposition 5.6 that the induced quotient topology on $\mathrm{S}_{R}^{\bullet}(W) /\left\langle\hat{A}^{2}+4 \hat{B} \hat{C}-1\right\rangle$ coincides with the quotient- $T_{R}$-topology under the above isomorphism.

So we would like to see that $\mathrm{S}_{R}^{\bullet}(W) /\left\langle\hat{A}^{2}+4 \hat{B} \hat{C}-1\right\rangle$ is homeomorphic to $\mathscr{R}_{\mathbb{C}^{2}} /\langle\hat{U} \hat{\bar{U}}+\hat{V} \hat{\bar{V}}-1\rangle$. To this end we define two maps $f: \mathrm{S}^{\bullet}(W) \rightarrow \mathscr{R}_{\mathbb{C}^{2}}$ and $g: \mathscr{R}_{\mathbb{C}^{2}} \rightarrow \mathrm{S} \bullet(W)$ by extending

$$
\begin{aligned}
f\left(\hat{A}^{\alpha} \hat{B}^{\beta} \hat{C}^{\gamma}\right) & =(\hat{U} \hat{\bar{U}}-\hat{V} \hat{\bar{V}})^{\alpha}(\hat{\bar{U}} \hat{V})^{\beta}(\hat{U} \hat{\bar{V}})^{\gamma} \\
g\left(\hat{U}^{\alpha} \hat{\bar{U}}^{\beta} \hat{V}^{\gamma} \hat{\bar{V}}^{\delta}\right) & =\left(\frac{1+\hat{A}}{2}\right)^{\alpha-\alpha \wedge \delta}\left(\frac{1-\hat{A}}{2}\right)^{\delta-\alpha \wedge \delta} \hat{B}^{\beta \wedge \gamma} \hat{C}^{\alpha \wedge \delta}
\end{aligned}
$$

linearly. Here we used the shorthand $m \wedge n=\min \{n, m\}$. It is easy to check that these maps descend to bijections on the quotients, so we shall be done if we can show that they are continuous. Set $d=\alpha+\beta+\gamma$, then

$$
\begin{aligned}
(C \mathfrak{p})_{R}\left(f\left(\hat{A}^{\alpha} \hat{B}^{\beta} \hat{C}^{\gamma}\right)\right) & =(C \mathfrak{p})_{R}\left(\sum_{i=0}^{\alpha}\left(\begin{array}{c}
\alpha \\
i
\end{array}\right)(\hat{U} \hat{\bar{U}})^{i}(-\hat{V} \hat{\bar{V}})^{\alpha-i}(\hat{\bar{U}} \hat{V})^{\beta}(\hat{U} \hat{\bar{V}})^{\gamma}\right) \\
& \leq \sum_{i=0}^{\alpha}\left(\begin{array}{c}
\alpha \\
i
\end{array}\right)((2 d) !)^{R} C^{2 d} \\
& =2^{\alpha}\left(\begin{array}{c}
2 d \\
d
\end{array}\right){ }^{R}(d !)^{2 R} C^{2 d} \\
& \leq 2^{d} 2^{2 d R}(d !)^{2 R} C^{2 d} \\
& =\left(2^{2 R+1} C^{2}\|\cdot\|_{1}^{\prime}\right)_{2 R}\left(\hat{A}^{\alpha} \hat{B}^{\beta} \hat{C}^{\gamma}\right)
\end{aligned}
$$

and the continuity of $f$ follows from Corollary 5.11. Similarly, setting $d^{\prime}=\alpha+\beta+\gamma+\delta$ and using that $\alpha+\gamma=\beta+\delta$ for elements of $\mathscr{R}_{\mathbb{C}^{2}}$ we obtain

$$
\begin{aligned}
\left(C\|\cdot\|_{1}^{\prime}\right)_{2 R}\left(g\left(\hat{U}^{\alpha} \hat{\bar{U}}^{\beta} \hat{V}^{\gamma} \hat{\bar{V}}^{\delta}\right)\right) & =\left(C\|\cdot\|_{1}^{\prime}\right)_{2 R}\left(\left(\frac{1+\hat{A}}{2}\right)^{\alpha-\alpha \wedge \delta}\left(\frac{1-\hat{A}}{2}\right)^{\delta-\alpha \wedge \delta} \hat{B}^{\beta \wedge \gamma} \hat{C}^{\alpha \wedge \delta}\right) \\
& \leq\left(C\|\cdot\|_{1}^{\prime}\right)_{2 R}\left(\hat{A}^{|\alpha-\delta|} \hat{B}^{\beta \wedge \gamma} \hat{C}^{\alpha \wedge \delta}\right) \\
& =\left(\left(d^{\prime} / 2\right) !\right)^{2 R} C^{d^{\prime} / 2} \\
& \leq\left(d^{\prime} !\right)^{R} C^{d^{\prime} / 2} \\
& =\left(C^{1 / 2} \mathfrak{p}\right)_{R}\left(\hat{U}^{\alpha} \hat{\bar{U}}^{\beta} \hat{V}^{\gamma} \hat{\bar{V}}^{\delta}\right) .
\end{aligned}
$$

Here we used that $\alpha+\delta-2 \alpha \wedge \delta=|\alpha-\delta|$ and that

$$
2(\beta \wedge \gamma+\alpha \wedge \delta+|\alpha-\delta|)=2 \beta \wedge \gamma+|\beta-\gamma|+2 \alpha \wedge \delta+|\alpha-\delta|
$$




$$
\begin{aligned}
& =\beta+\gamma+\alpha+\delta \\
& =d^{\prime} .
\end{aligned}
$$

We want to finish this subsection with the following easy observation.

Proposition 5.15 The quotient- $T_{R}$-topology and the reduction- $T_{R}$-topology are Hausdorff.

ProOF: It suffices to prove that the ideal divided out is closed. For the quotient- $T_{R}$-topology it is the intersection of the kernels of all evaluation functionals at points of the 2-sphere and these functionals are continuous with respect to any $T_{R}$-topology.

\subsection{Continuity in the reduction and quotient- $T_{R}$-topologies}

In this section we will prove the continuity of $*_{\hbar}$ with respect to the reduction- $T_{R}$-topology, using the approach of Alekseev-Lachowska. We will outline how the approach of Karabegov can be used to prove continuity with respect to the quotient- $T_{R}$-topology and prove that the star product depends holomorphically on $\hbar$ on the completion.

Theorem 5.16 The star product $*_{\hbar}$ on $\mathbb{S}_{\lambda}^{2}$ is continuous with respect to the reduction- $T_{R}$-topology for $R \geq 0$ if $\frac{\lambda}{\hbar} \notin \mathbb{N}$.

ProOF: Recall from Lemma 5.8 that both $\left(X^{\text {left }}\right)^{n}$ and $\left(Y^{\text {left }}\right)^{n}$ vanish on monomials of degree $\leq n-1$ and neither of them raises the degree of a monomial. Set $p_{\alpha \beta \gamma \delta}=\hat{U}^{\alpha} \hat{\bar{U}}^{\beta} \hat{V}^{\gamma} \hat{\bar{V}}^{\delta}$ for $\alpha, \beta, \gamma, \delta \in \mathbb{N}_{0}$ and let $d=\alpha+\beta+\gamma+\delta$. Note that $\left(Y^{\text {left }}\right)^{n} p_{\alpha \beta \gamma \delta}\left(X^{\text {left }}\right)^{n} p_{\alpha^{\prime} \beta^{\prime} \gamma^{\prime} \delta^{\prime}}$ is a sum of at most $\frac{d !}{(d-n) !} \frac{d^{\prime} !}{\left(d^{\prime}-n\right) !}$ such monomials of degree $d+d^{\prime}$. So for $C \geq 1$ we obtain that

$$
\begin{aligned}
(C \mathfrak{p})_{R}\left(p_{\alpha \beta \gamma \delta} * \mathscr{P} \hbar p_{\alpha^{\prime} \beta^{\prime} \gamma^{\prime} \delta^{\prime}}\right) & =(C \mathfrak{p})_{R}\left(\sum_{n=0}^{\infty} \frac{(-1)^{n}}{n ! \frac{\lambda}{\hbar}\left(\frac{\lambda}{\hbar}-1\right) \ldots\left(\frac{\lambda}{\hbar}-(n-1)\right)}\left(Y^{\text {left }}\right)^{n} p_{\alpha \beta \gamma \delta}\left(X^{\text {left }}\right)^{n} p_{\alpha^{\prime} \beta^{\prime} \gamma^{\prime} \delta^{\prime}}\right) \\
& \leq \sum_{n=0}^{\min \left\{d, d^{\prime}\right\}} \frac{1}{(n !)^{2} C_{-}^{n}} \frac{d !}{(d-n) !} \frac{d^{\prime} !}{\left(d^{\prime}-n\right) !}\left(d+d^{\prime}\right) !{ }^{R} C^{d+d^{\prime}} \\
& \leq 2^{\min \left\{d, d^{\prime}\right\}} C_{-}^{-\min \left\{d, d^{\prime}\right\}} 2^{d} 2^{d^{\prime}}\left(2^{d+d^{\prime}} d ! d^{\prime} !\right)^{R} C^{d+d^{\prime}} \\
& \leq\left(2^{2+R} C C_{-}\right)^{d}(d !)^{R}\left(2^{2+R} C C_{-}\right)^{d^{\prime}}\left(d^{\prime} !\right)^{R} \\
& =\left(2^{2+R} C C_{-} \mathfrak{p}\right)_{R}\left(p_{\alpha \beta \gamma \delta}\right)\left(2^{2+R} C C_{-} \mathfrak{p}\right)_{R}\left(p_{\alpha^{\prime} \beta^{\prime} \gamma^{\prime} \delta^{\prime}}\right) .
\end{aligned}
$$

By using Corollary 5.11 (or a similar version for maps with two arguments) the continuity of $*_{\hbar}^{\mathscr{P}}$ with respect to the $T_{R^{-}}$-topology induced by $\mathfrak{p}$ on $\mathscr{P}_{\mathbb{C}^{2}}$ follows and thus the continuity of $*_{\hbar}$ with respect to the reduction- $T_{R}$-topology.

The following theorem is obvious because of Proposition 5.14. However, it can also be proved independently by deriving a formula for $*_{\hbar}$ that only uses $\mathscr{R}_{\mathrm{SU}(2)}$. (Note that $p_{W, \bar{W}}:=Y^{\text {left }} W \cdot X^{\text {left }} \bar{W}$ is an element of $\mathscr{R}_{\mathrm{SU}(2)}$ for $W \in\{U, V\}, \bar{W} \in\{\bar{U}, \bar{V}\}$, so the $p_{W, \bar{W}}$ can be used to replace the left invariant vector fields applied to polynomials.)

Theorem 5.17 The star product $*_{\hbar}$ on $\mathbb{S}_{\lambda}^{2}$ is continuous with respect to the quotient- $T_{R}$-topology for $R \geq 0$ if $\frac{\lambda}{\hbar} \notin \mathbb{N}$.

According to Theorem 5.16 the star product $*_{\hbar}$ is continuous on $\operatorname{Pol}\left(\mathbb{S}_{\lambda}^{2}\right)$ endowed with the reduction$T_{R}$-topology for $R \geq 0$. Extend $*_{\hbar}$ to the completion $\widehat{\mathrm{Pol}}_{R}\left(\mathbb{S}_{\lambda}^{2}\right)$, still denoted by $*_{\hbar}$. 
Lemma 5.18 For all $x \in \mathbb{S}_{\lambda}^{2}$ the evaluation functional $\mathrm{ev}_{x}^{\mathbb{S}}: \operatorname{Pol}\left(\mathbb{S}_{\lambda}^{2}\right) \rightarrow \mathbb{C}, p \mapsto p(x)$ is continuous in the reduction- $T_{R}$-topology.

Proof: Take any $k=\left(\begin{array}{c}u \\ v \\ \bar{u}\end{array}\right) \in K$ with $\psi_{\mu}(k)=x$. Then $\operatorname{ev}_{x}^{\mathbb{S}}$ is the induced quotient map of the continuous map ev $(u, v)$ and therefore continuous.

Recall that $\Omega=\left\{0, \lambda, \frac{\lambda}{2}, \frac{\lambda}{3}, \frac{\lambda}{4}, \ldots\right\}$.

Theorem 5.19 For fixed $p, q \in \widehat{\operatorname{Pol}}_{R}\left(\mathbb{S}_{\lambda}^{2}\right)$ and $x \in \mathbb{S}_{\lambda}^{2}$ the function

$$
\begin{aligned}
\mathbb{C} \backslash \Omega & \longrightarrow \mathbb{C} \\
\hbar & \longmapsto p * \hbar q(x)
\end{aligned}
$$

is holomorphic in $\hbar$.

Proof: Choose sequences of polynomials $p_{n}, q_{n} \in \operatorname{Pol}\left(\mathbb{S}_{\lambda}^{2}\right)$ with $p_{n} \rightarrow p$ and $q_{n} \rightarrow q$ for $n \rightarrow \infty$. The star product $*_{\hbar}$ is continuous, so we also have $p_{n} *_{\hbar} q_{n} \rightarrow p *_{\hbar} q$. Continuity of ev ${ }_{x}^{\mathbb{S}}$ implies that $p_{n} *_{\hbar} q_{n}(x) \rightarrow p *_{\hbar} q(x)$. Since by Theorem 2.9 all maps $\hbar \mapsto p_{n} *_{\hbar} q_{n}(x)$ are rational with finitely many poles in $\Omega$, they are in particular holomorphic on $\mathbb{C} \backslash \Omega$. So it suffices to prove that $p_{n} *_{\hbar} q_{n}(x) \rightarrow p *_{\hbar} q(x)$ locally uniformly in $\hbar$. But Lemma 5.12 gives a locally uniform estimate, so all estimates in the previous section are locally uniformly in $\hbar$, so by applying $\mathrm{ev}_{x}^{\mathbb{S}}$ the result follows.

Remark 5.20 (Continuity by brute force) We remark that Theorem 5.17 can be also proved in a more direct but complicated way. Here we only sketch the main steps and refer to [37, Chapter 4] for the details. First, observe that one can calculate the holomorphic derivatives $\xi_{W} Z^{b}$ for $W, Z \in$ $\{H, X, Y\}$ explicitly. This calculation shows that one can extend them uniquely to polynomials on $\mathfrak{s u}_{2}$ in such a way that $\xi_{W} Z^{b}=\frac{i}{\lambda} W^{b} Z^{b}+$ lower order terms. Using this extension, one can define

$$
\hat{L}^{(\hbar)}: \mathrm{S} \bullet\left(\mathfrak{s l}_{2}(\mathbb{C})\right) \rightarrow \operatorname{Pol}\left(\mathfrak{s u}_{2}\right)
$$

in the same way as $L^{(\hbar)}$ before, but using the derivatives $\xi_{W} Z^{\mathrm{b}}$ from above and interpreting $f_{Z}=Z^{\mathrm{b}}$ as an element of $\operatorname{Pol}\left(\mathfrak{s u}_{2}\right)$. It turns out that $\hat{L}^{(\hbar)}$ is invertible (away from the poles).

Lemma 5.21 Karabegov's star product on $\operatorname{Pol}\left(\mathbb{S}_{2}^{\lambda}\right)$ can be written as

$$
\left.\left.f\right|_{\mathbb{S}_{\lambda}^{2}} *_{\hbar} g\right|_{\mathbb{S}_{\lambda}^{2}}=\left.\hat{L}^{(\hbar)}\left(\left(\hat{L}^{(\hbar)}\right)^{-1}(f) *_{\mathrm{Gutt}, 1}\left(\hat{L}^{(\hbar)}\right)^{-1}(g)\right)\right|_{\mathbb{S}_{\lambda}^{2}}
$$

for $f, g \in \operatorname{Pol}\left(\mathfrak{s u}_{2}\right)$. Here we denoted by $*_{\text {Gutt }, 1}$ the Gutt star product for $\hbar=1$ viewed as an associative deformation of the symmetric algebra $\mathrm{S}^{\bullet}\left(\mathfrak{s l}_{2}(\mathbb{C})\right)$.

To obtain continuity estimates we need explicit formulas for $\hat{L}^{(\hbar)}$ and its inverse, see [37, Lemma 4.2.16]. These formulas and the observation that we get $\mathscr{A}_{\hbar}=\operatorname{Pol}\left(\mathbb{S}_{\lambda}^{2}\right)$ whenever $\lambda / \hbar \notin \mathbb{N}_{0}$ allow us to prove the following proposition.

Proposition 5.22 For $R \geq 0$ and $\lambda / \hbar \notin \mathbb{N}_{0}$ the map $\hat{L}^{(\hbar)}$ is a homeomorphism from $\mathrm{S}_{R+1}^{\bullet}\left(\mathfrak{s l}_{2}(\mathbb{C})\right.$ ) to $\mathrm{S}_{R}^{\bullet}\left(\mathfrak{s l}_{2}(\mathbb{C})^{*}\right)$.

Proof: See [37, Theorem 4.2.20].

The continuity of Karabegov's star product $*_{\hbar}$ on $\mathbb{S}_{\lambda}^{2}$ with respect to the quotient- $T_{R}$-topology (if $\lambda / \hbar \notin \mathbb{N}_{0}$ ) follows from (5.18) and Proposition 5.22 since the Gutt star product is continuous with respect to the $T_{R}$-topology on $\mathrm{S}^{\bullet}\left(\mathfrak{s l}_{2}(\mathbb{C})\right)$ for $R \geq 1$ according to [18]. 


\section{References}

[1] Alekseev, A., Lachowska, A.: Invariant *-products on coadjoint orbits and the Shapovalov pairing. Comment. Math. Helv. 80 (2005), 795-810.

[2] Bayen, F., Flato, M., Frønsdal, C., Lichnerowicz, A., Sternheimer, D.: Deformation Theory and Quantization. Ann. Phys. 111 (1978), 61-151.

[3] Beiser, S., Römer, H., Waldmann, S.: Convergence of the Wick Star Product. Commun. Math. Phys. 272 (2007), 25-52.

[4] Beiser, S., Waldmann, S.: Fréchet algebraic deformation quantization of the Poincaré disk. Crelle's J. reine angew. Math. 688 (2014), 147-207.

[5] Bertelson, M., Cahen, M., Gutt, S.: Equivalence of Star Products. Class. Quant. Grav. 14 (1997), A93-A107.

[6] Bieliavsky, P. Quantum differential surfaces of higher genera. ArXiv e-prints (2017).

[7] Bieliavsky, P., Gayral, V.: Deformation Quantization for Actions of Kählerian Lie Groups. Memoirs of the American Mathematical Society 236.1115 (2015).

[8] Bordemann, M., Brischle, M., Emmrich, C., Waldmann, S.: Phase Space Reduction for Star Products: An Explicit Construction for $\mathbb{C} P^{n}$. Lett. Math. Phys. 36 (1996), 357-371.

[9] Bordemann, M., Brischle, M., Emmrich, C., Waldmann, S.: Subalgebras with converging star products in deformation quantization: An algebraic construction for $\mathbb{C} P^{n}$. J. Math. Phys. 37 (1996), 6311-6323.

[10] Bordemann, M., Forger, M., Römer, H.: Homogeneous Kähler manifolds: paving the way towards new supersymmetric sigma models. Commun. Math. Phys. 102 (1986), 605-617.

[11] Bordemann, M., Waldmann, S.: A Fedosov Star Product of Wick Type for Kähler Manifolds. Lett. Math. Phys. 41 (1997), 243-253.

[12] Cahen, M., Gutt, S., Rawnsley, J.: Quantization of Kähler Manifolds I: Geometric Interpretation of Berezin's Quantization. J. Geom. Phys. 7 (1990), 45-62.

[13] Cahen, M., Gutt, S., Rawnsley, J.: Quantization of Kähler Manifolds. II. Trans. Am. Math. Soc. 337.1 (1993), 73-98.

[14] Cahen, M., Gutt, S., Rawnsley, J.: Quantization of Kähler Manifolds. III. Lett. Math. Phys. 30 (1994), 291-305.

[15] Cahen, M., Gutt, S., Rawnsley, J.: Quantization of Kähler Manifolds. IV. Lett. Math. Phys. 34 (1995), 159-168.

[16] Calaque, D., NÄF, F.: A trace formula for the quantization of coadjoint orbits. ArXiv e-prints (mar 2014).

[17] DeWilde, M., Lecomte, P. B. A.: Existence of Star-Products and of Formal Deformations of the Poisson Lie Algebra of Arbitrary Symplectic Manifolds. Lett. Math. Phys. 7 (1983), 487-496.

[18] Esposito, C., Stapor, P., Waldmann, S. Convergence of the Gutt Star Product. J. Lie Theory 27 (2017), $579-622$.

[19] Fedosov, B. V.: A Simple Geometrical Construction of Deformation Quantization. J. Diff. Geom. 40 (1994), 213-238.

[20] Gutr, S.: An Explicit *-Product on the Cotangent Bundle of a Lie Group. Lett. Math. Phys. 7 (1983), $249-258$.

[21] Karabegov, A. V.: Deformation Quantization with Separation of Variables on a Kähler Manifold. Commun. Math. Phys. 180 (1996), 745-755.

[22] Karabegov, A. V.: Berezin's quantization on flag manifolds and spherical modules. Trans. Amer. Math. Soc. 350.4 (1998), 1467-1479.

[23] Karabegov, A. V.: Pseudo-Kähler Quantization on Flag Manifolds. Commun. Math. Phys. 200 (1999), 355-379.

[24] Kirillov, A.: Unitary representations of nilpotent Lie groups. Russian Mathematical Surveys 17.4 (1962), 53.

[25] Knapp, A. W.: Lie Groups Beyond an Introduction. Progress in Mathematics. Birkhäuser Basel, 2. edition, 2002.

[26] Kontsevich, M.: Deformation Quantization of Poisson manifolds. Lett. Math. Phys. 66 (2003), $157-216$.

[27] Kraus, D., Roth, O., Schötz, M., Waldmann , S.: A Convergent Star Product on the Poincaré Disc . ArXiv:1803.02763 (2018).

[28] Marsden, J. E., Ratiu, T. S.: Introduction to Mechanics and Symmetry. Texts in applied mathematics no. 17. Springer-Verlag, New York, Heidelberg, 1999. 
[29] Moreno, C., Ortega-Navarro, P.: *-Products on $D^{1}(\mathbb{C}), S^{2}$ and Related Spectral Analysis. Lett. Math. Phys. 7 (1983), 181-193.

[30] Müller-Bahns, M. F., Neumaier, N.: Invariant Star Products of Wick Type: Classification and Quantum Momentum Mappings. Lett. Math. Phys. 70 (2004), 1-15.

[31] Natsume, T., Nest, R.: Topological Approach to Quantum Surfaces. Commun. Math. Phys. 202 (1999), 65-87.

[32] Natsume, T., Nest, R., Peter, I.: Strict Quantizations of Symplectic Manifolds. Lett. Math. Phys. 66 (2003), 73-89.

[33] Nest, R., Tsygan, B.: Algebraic Index Theorem. Commun. Math. Phys. 172 (1995), 223-262.

[34] Neumaier, N.: Universality of Fedosov's Construction for Star Products of Wick Type on Pseudo-Kähler Manifolds. Rep. Math. Phys. 52 (2003), 43-80.

[35] Rieffel, M. A.: Deformation quantization for actions of $\mathbb{R}^{d}$. Mem. Amer. Math. Soc. 106.506 (1993), 93 pages.

[36] Rieffel, M. A. Questions on quantization. Contemporary Mathematics 228 (1998).

[37] Schmitt, P.: Convergent Star Products on Coadjoint Orbits. Master thesis (2017).

[38] Sснӧтz, M., Waldmann, S.: Convergent star products for projective limits of Hilbert spaces. Journal of Functional Analysis 274.5 (2018), 1381 - 1423.

[39] Waldmann, S.: A nuclear Weyl algebra. J. Geom. Phys. 81 (2014), 10-46. 